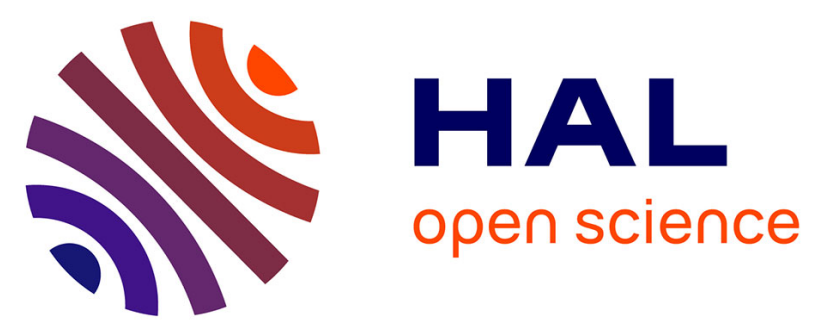

\title{
A Multicriteria Decision Support System using a Possibility Representation for Managing Inconsistent Assessments of Experts Involved in Emergency Situations
}

Abdelhak Imoussaten, Jacky Montmain, Gilles Mauris

\section{To cite this version:}

Abdelhak Imoussaten, Jacky Montmain, Gilles Mauris. A Multicriteria Decision Support System using a Possibility Representation for Managing Inconsistent Assessments of Experts Involved in Emergency Situations. International Journal of Intelligent Systems, 2014, 29 (1), pp.50-83. 10.1002/int.21627 . hal-00944146

\author{
HAL Id: hal-00944146 \\ https://hal.science/hal-00944146
}

Submitted on 26 May 2021

HAL is a multi-disciplinary open access archive for the deposit and dissemination of scientific research documents, whether they are published or not. The documents may come from teaching and research institutions in France or abroad, or from public or private research centers.
L'archive ouverte pluridisciplinaire HAL, est destinée au dépôt et à la diffusion de documents scientifiques de niveau recherche, publiés ou non, émanant des établissements d'enseignement et de recherche français ou étrangers, des laboratoires publics ou privés. 


\title{
A Multicriteria Decision Support System using a Possibility Representation for Managing Inconsistent Assessments of Experts Involved in Emergency Situations
}

\author{
Abdelhak Imoussaten, ${ }^{1}$ Jacky Montmain, ${ }^{1}$ Gilles Mauris ${ }^{2, *}$ \\ ${ }^{1}$ LGI2P_Ecole des Mines d'Alès, Site EERIE-Parc Scientifique G. Besse \\ 30035, Nîmes Cedex, France \\ 2LISTIC_Polytech' Annecy-Chambéry, Université de Savoie, BP 80439 74944, \\ Annecy-le-Vieux, France
}

\begin{abstract}
Within an emergency unit, the head manager is required to make difficult decisions based on experts' assessments of many criteria, including personal injuries, environmental impacts, and economic and media consequences. Uncertainty in this collective assessment is related to the multiplicity of experts' points of view and imprecise assessments. We are proposing a decision support system derived from a situation-awareness model, generalized herein to the case of multiple actors. It is able of representing, merging, and aggregating expert assessments. Imprecise criteria assessments are first represented by intervals and then merged in the form of a possibility distribution that keeps track of all the information provided, that is, without any loss of information. Next, a Choquet integral based aggregation is carried out to consider the relative importance of criteria and interactions between criteria in the overall assessment of the foreseeable alternatives to get out of the crisis. Finally, a determination of the contributions of each criterion assessment uncertainty to the overall assessment uncertainty provides useful information to the head manager in controlling the decision deliberation by reducing the inconsistent points in the experts' assessments. The proposals are applied to the emergency issues resulting from a traffic accident occurring at a grade crossing.
\end{abstract}

\section{INTRODUCTION}

Emergency situations, regardless of their origin (e.g., natural hazard, infectious disease, industrial incident, and traffic accident, etc.), necessitate a coherent and effective emergency-management approach that involves complex decision making. ${ }^{1}$ In such a context, one key issue is to provide the emergency team with a computerized framework ${ }^{2}$ dedicated to:

\footnotetext{
*Author to whom all correspondence should be addressed; e-mail: gilles.mauris@ univ-savoie.fr.
} 
1. define the relevant dynamic response to the emergency situation as a collaborative process;

2. coordinate partners' actions through their information systems, in accordance with the previously established collaborative process;

3. ensure the temporal relevance of the reaction as the situation evolves.

According to Refs. 3-5 and 1, the decision-making process in an emergency context is altered by the following specificities: insufficient or excess information, inconsistent information, complexity, threat, surprise, and a potential conflict between heterogeneous actors. Consequently, the critical issues associated with an emergency situation (such as people's lives and irreversible effects) imply a fast and efficient decision-making process, while the specificities of the emergency situation (partial knowledge and a stressful environment) require an original and dedicated decision process. Moreover, decisions made in an emergency unit result from deliberation under pressure, wherein inconsistent or contradictory information exchanges disturb communication and coordination within the unit and ultimately slow the decision process. In this regard, Ref. 6 proposes a cooperative situation assessment in a maritime scenario: their solution, which optimizes the shared amount of knowledge about the situation, relies on a distributed algorithm for situation assessment, which solves disagreements among agents by using sequences of one-to-one interactions.

Finally, crisis-management decisions are driven by many criteria, including personal injuries, environmental impacts, and economic and media consequences. Accordingly, the development and application of team-wide multicriteria decision support systems (DSS) could prove extremely valuable in support of emergencymanagement decisions. ${ }^{2,7-9}$ From this point of view, we present herein a system dedicated to road traffic accidents on the basis of a situation-awareness model, ${ }^{10,11}$ with a focus on the multicriteria decision aids. In emergency situations, many conflicting objectives to select the most efficient option to get out of the crisis must in fact be resolved and priorities must be set, while the various perspectives of many different stakeholder groups must be merged into some kind of consensus. Multicriteria decision aiding can thus help ensure transparency during the decision-making process. A Choquet integral (CI)-based aggregation ${ }^{12,13}$ is considered effective in capturing the relative importance of criteria and interactions between criteria within the overall aggregated assessment of competing alternatives to correct the situation.

The handling of uncertainties is a fundamental part of such an effective multicriteria decision-making tool, although, in practice, it is important not to overload the users of a decision system with excessive information on uncertainties. Success of this step requires an understandable representation of uncertainties generated by experts in the aggregated decision-making process and the control of the debate. Our proposal, therefore, is based on a possibility/fuzzy representation ${ }^{14}$ of experts' assessments and their associated summary indicators, which are better adapted than probability and are simpler than the Dempster-Shafer theory ${ }^{15-17}$ in guiding the participating decision makers and stakeholders during the team-moderated discussion leading to the final multicriteria decision.

This paper will be organized as follows. Section 2 will present the specificities of situation awareness (SA) and decision making in an emergency management 


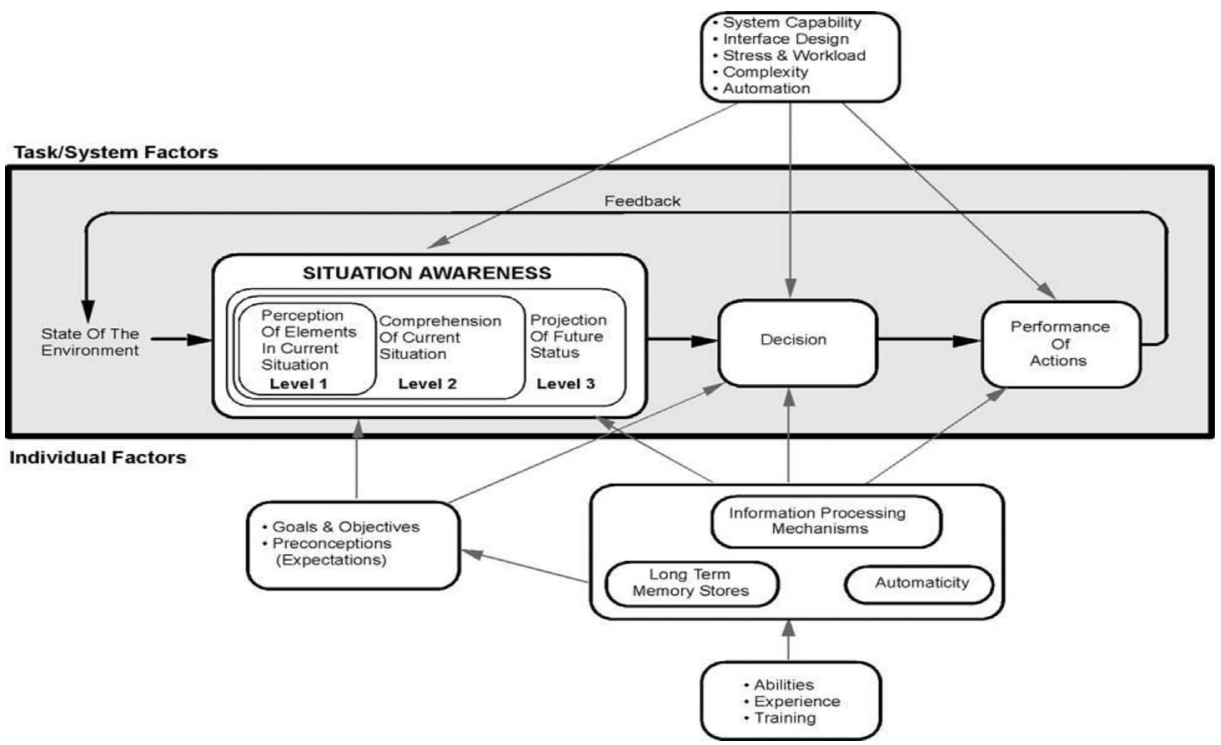

Figure 1. Situation awareness and decision making. ${ }^{19}$

context. Section 3 will describe the article's main contributions relative to the representation, merging, and aggregation of experts' assessments and to the way the proposed uncertainty indicators are used to settle deliberation issues. A road traffic accident case study will highlight the interests of these proposals in Section 4.

\section{SITUATION AWARENESS AND DECISION MAKING IN AN EMERGENCY MANAGEMENT CONTEXT: ISSUES AND SPECIFICITIES}

Situation awareness involves becoming aware of all that is happening in the vicinity to understand how information, events, and one's own actions will alter goals and objectives, both immediately and in the near future. A lack of SA or an inadequate SA has been identified as one of the primary factors in accidents attributed to human error. ${ }^{18}$ In fact, SA-related cognitive mechanisms (perception, comprehension, and projection into the future), decision making, and performance assessment are all embedded into a cognitive feedback loop ${ }^{19}$ (see Figure 1).

The perceptual level 1 involves the detection, recognition, and identification of elements that define a specific situation. Perceptual SA relies on the available sensory information (e.g., from sensors) as well as the operator's/decision maker's prior knowledge (e.g., object patterns/diagrams activated by memory) to identify individual situation elements and object groups. Comprehension level 2 reflects an understanding of the current state of affairs and involves making inferences about activities in the current situation. As such, the comprehension level maps perception 
products into object functions. Finally, projection level 3 consists of interpretations concerning the trajectory of the situation based on the output of comprehension SA and prior knowledge. ${ }^{20}$

In emergency situations, decision making in the management unit features three main specificities:

i. Multiple actors and multiple criteria: An initial significant characteristic of an emergency management unit pertains to the deep-rooted structural heterogeneity of its partners. Depending on the specificities of the emergency situation (geographical location, nature of the problem, stakes involved, and seriousness, etc.), the emergency unit may be composed, for example, of representatives wielding national authority, local stakeholders, different branches of the military, fire fighters, police officers, medical teams, public works departments, and nongovernmental organizations. All these actors may consider differently the SA situation, its consequences, stakes, and solution criteria. According to Ref. 21, even if actors seek to obtain a collective perception of the situation (through "sense-making"), they might also retain their own vision and assessments of the emergency situation (based on their particular field of business, culture, and ability to understand or contextualize information). Decisions involved in the emergency situation also include many viewpoints, all within an evolving social or environmental SA context, and are, therefore, essentially multicriteria in nature and dependent upon human, social, technical, regulatory, safety, and financial aspects. Comparing competing alternatives to relevantly correct the situation whose strengths or weaknesses relate to multiple objectives of interest to the decision maker is thus a key issue in reaching a final decision.

ii. Uncertainty context: A specificity regarding the mental SA representation of an emergency situation is its development from very limited and informal material. While in other contexts, such a mental representation might be based on the actual subject itself (due to its accessibility), in an emergency context, the subject becomes extremely blurred and the mental image can only be drawn on the basis of fragmented information and not directly from the situation itself. Such an intermediate model (consisting of fragmented knowledge gathered from the emergency field and provided to the emergency unit) implies an additional level of abstraction along with all the associated cultural and business interpretations.

iii. Expert assessments: Decision making in an emergency management unit implies taking into account experts' points of view in a relevant manner. Depending on the type of decision to be made (i.e., what are the possible options for resolving the crisis, which evaluation criteria are applicable to these options, etc.), only a few members of the management unit might actually be able to provide relevant assessments. ${ }^{22}$ The perception might neither be accurate nor objective, and even experts' assessments may prove to be controversial. The SA perception phase must therefore deal with imprecise, uncertain, and conflicting assessments. Furthermore, security, administrative, or military authorities entrust experts to perform the comprehension phase, in which case 
experts must debate and explain any observed disagreements. These conflicts in experts' judgment constitute an additional source of uncertainty in the SA assessment. No point of view can be ignored since any given expert may be alone in fully realizing the genuine consequences of the crisis.

In summary, due to (1) the large cultural heterogeneity of emergency management unit actors, (2) the extremely limited level of knowledge available within the emergency management unit, and (3) the need to consider various experts' assessments in a nondemocratic (or nonsmoothing) approach, the decision-making domain in emergency management comprises some very specific components. Accordingly, this paper proposes a mathematical model for managing the SA assessment and option selection phases when the assessment is being performed by a group of experts. Endsley's model will thus be completed herein using an internal cognitive feedback loop, whose control variable is the level of uncertainty in experts' assessments and whose aim is to efficiently manage the experts' debate in emergency situations (see the internal loop on the SA activity in Figure 1). The experts are indeed required to debate and explain their assessments to clarify the situation for the management unit's head manager; hence, the collective decision-making process must keep the full array of expert assessments along with their uncertainties so as to identify areas of dispute in the experts' debate and then efficiently control them relative to these uncertainties. Finally, the cognitive feedback loop of our model must be based on an easily understandable model of uncertainty management for interactive and comprehension purposes by the emergency unit.

\section{REPRESENTING, MERGING, AGGREGATING, AND COMPARING EXPERT ASSESSMENTS}

In an emergency management unit, the role of an expert in collective decision making is to provide a priori reliable assessments for those criteria lying within his area of expertise. The resulting questions are: (1) how to merge assessments on one criterion without a loss of relevant information and without neglecting anyone's point of view? (2) how to aggregate experts' assessments when forecasts are to be produced with multiple criteria? (3) and how to organize the deliberation process to resolve the most highly disputed points among experts? According to this view, the DSS proposed here aims to support the emergency unit's head to select the most efficient alternative to get out of the crisis in merging and aggregating the experts' assessments without losing any indications regarding possible dispute issues among experts. Then, the choice of the aggregation operator and the way uncertainty is handled in the merging process are crucial. As the aggregated assessment of experts' collective views must keep track of all experts' assessments, similarity aggregation method (SAM) ${ }^{23,24}$ must be dismissed here because in the SAM approach the weight of an expert's assessment should depend on this expert's level of agreement with respect to other experts. Indeed, in our framework, to make a reliable decision, the head manager of the crisis unit must verify the origin of all discrepancies (incorrect, imprecise, or uncertain data, and misinterpretations of experts' assessments, etc.) 
and then attempt to minimize them by reorienting the debate on the competing points. Uncertainties in the experts' assessments must therefore be explicitly represented and propagated into the merging and aggregation processes. With this aim, it will now be shown that the possibility theory provides some relevant tools. ${ }^{25}$ Afterwards, a quantification of the influence of criteria assessment uncertainties on the overall assessment uncertainty will be proposed to provide the head manager with a means to efficiently guide the debate and converge on the most consensual decision as quickly as possible. An efficient support will thus be provided to control both the SA assessment step, and the points experts must focus on first to quickly reach a decision-making situation.

\subsection{Framework of the Proposed DSS and Associated Notations}

To assist the emergency unit head manager in selecting an alternative from several decision criteria, a global evaluation is conducted with an adequate aggregation of all expert assessments. The proposed merging process that combines imprecise assessments relative to a given criterion is based on possibility theory, ${ }^{14}$ and moreover, merges these assessments without any loss of information; it computes the group assessment for each criterion. The aggregation process that combines these group assessments relative to each criterion is performed by the head manager: experts provide all necessary information or evidence to support the emergency management unit head manager, even though this manager is the only one to decide. The aggregation model represents the decision maker's decision strategy and, as such, must be clear enough to be easily understood; it is expected to be used to explain or justify any emergency unit decision. From this perspective, the CI-based aggregation, which accounts for interactions between criteria while maintaining the compromise aspect of the conventional average, offers an interesting tool that has already been used by several authors. ${ }^{26}$ In dealing with uncertainty due to all actors involved in the emergency unit, ${ }^{27}$ it is proposed to use possibility distributions to gather and merge all expert assessments with respect to a criterion. This procedure is motivated by the nature as well as the availability of the data: the evaluations of alternatives stemming from our application in essence correspond to imprecise human assessments by experts and are far from being precise numerical assessments. Moreover, few expert evaluations are available, which further complicates any reliable identification of probability distributions. ${ }^{28}$ Possibility theory also provides an understandable representation of uncertainties that can be propagated into the decision-making process, while explanation capabilities may be proposed. ${ }^{29}$

In summary, we will detail in the following subsections the four key processes involved, that is:

- Merging process: Construction of possibility distributions to merge experts' opinions with respect to each criterion. Our main contribution at this level is to extend existing methods for the case of consistent opinions (unimodal distributions of assessments) to the case when opinions are inconsistent (multimodal distributions);

- Aggregation process: The aggregation of criterion possibility distributions using the CI. Our main contribution at this level consists of establishing a linear relationship between the partial and aggregated evaluations, thanks to a suitable method of possibility distribution aggregation; 
- Description process: The characterization of possibility distributions by defining descriptive indicators that simplify the quantitative interpretation. For this purpose, key indicators, such as location and uncertainty indicators, will be provided;

- Comparison process: Finally, the tools developed will be used for analyzing the experts' opinions. Whenever divergent assessments are provided for a key criterion, the debate is centered on this criterion so that all disputed points are cleared before making the decision.

\subsubsection{Notations}

Let's consider a set of $l$ possible alternatives $\left\{A l^{q}, 1 \leq q \leq l\right\}$ for a given decision-making problem. Each alternative $A l^{q}$ is evaluated according to $n$ criteria. Let's also consider a data base of $N E$ experts $\left\{E v l^{j}, 1 \leq j \leq N E\right\}$. Assessments of experts express imprecise degrees of satisfaction and are represented by intervals in our framework. Let $I_{i}^{j, q}$ be the assessment given by expert $E v l^{j}$ according to criterion $i$ for alternative $A l^{q}$. As mentioned above, possibility theory is applied to take into account the variability of assessments provided by all experts for a given alternative $A l^{q}$. Possibility distributions $\pi_{i}^{q}$ are thus built from the merging of imprecise assessments $I_{i}^{j, q}$, with respect to criterion $i$ : the merging process yields the possibility evaluation of alternative $A l^{q}$ with respect to criterion $i$. Once $\pi_{i}^{q}(i=1, \ldots, n)$ have been built for each criterion, they can then be aggregated to compute the overall possibility evaluation $\pi_{a g}^{q}$ of alternative $A l^{q}$.

\subsection{Representation of Uncertainties: Belief Functions and Possibility Distributions}

Because of limited perception capabilities and variability of the considered phenomenon, it is quite common for an expert to provide assessments by means of intervals rather than specific unique values. The evidence theory ${ }^{30,31}$ (or belief function theory) is an appropriate framework to represent such a set of intervals. Indeed, belief functions appear to play a pivotal role since they generalize both the probability and possibility distributions ${ }^{25}$ and have been used in the emergency decision-making context. ${ }^{9}$ Yet, they generally lead to rather complex processing; consequently, hereafter we will be using the belief-function framework, though we will ultimately represent assessments by possibility distributions given their good approximation of belief functions. Also, possibility functions are appealing from an interpretation point of view in collecting confidence intervals as well as a computational point of view. ${ }^{32}$

\subsubsection{Possibility Theory}

Let $\Omega$ represent a universal set of elements $\omega$ under considerations that are assumed to be finite and let $2^{\Omega}$ represent the power set of $\Omega$. A possibility distribution $\pi$ is a normalized function $\pi: \Omega \rightarrow[0,1]$ (i.e., $\exists \omega \in \Omega$, such that $\pi(\omega)=1$ ). (see Figure 2 for an example of $\pi$ ). From $\pi$, possibility and necessity measures are respectively defined as: $\Pi(A)=\sup _{\omega \in A} \pi(\omega)$ and $N(A)=1-\Pi\left(A^{c}\right) \forall A \subseteq \Omega$. $\Pi(A)$ 


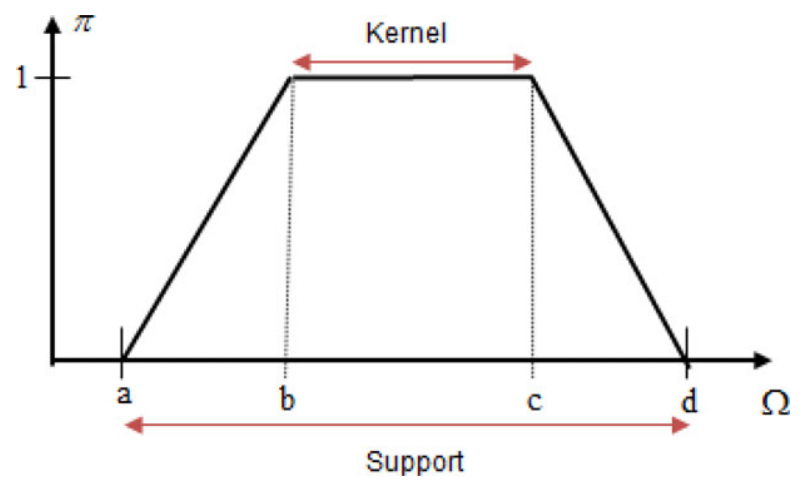

Figure 2. Trapezoidal representation of a possibility distribution.

quantifies to what extent the event $A$ is plausible while $N(A)$ quantifies the certainty of $A$. An $\alpha$-cut of possibility distribution $\pi$ is the set $E_{\alpha}(\pi)=\{\omega \in \Omega / \pi(\omega) \geq \alpha\}$, $\alpha \in] 0,1]$.

\subsubsection{Evidence Theory}

The evidence theory shall now be formulated by the basic belief assignment (bba) $m$ defined from $2^{\Omega}$ to $[0,1]$, such that: $\sum_{A \subset \Omega} m(A)=1$ and $m(\emptyset)=0$. Elements $E$ of $2^{\Omega}$ such that $m(E)>0$ are called focal elements and their set is denoted by $\mathbb{F}$. The $b b a m$ can be represented by two measures: the belief function $\operatorname{Bel}(A)=\sum_{\{E \in \mathbb{F} / A \supseteq E\}} m(E), \forall A \subseteq \Omega$ and the plausibility function $P l(A)=\sum_{\{E \in \mathbb{F} / A \cap E \neq \emptyset\}} m(E), \forall A \subseteq \Omega$.

As a matter of fact, evidence theory is nothing more than the rules of conventional probability theory applied to imprecise statements, that is, the focal elements instead of precise point statements. The belief measure (also called credibility measure) of event $A$ combines the various evidences in support of $A$, that is, whose occurrences imply the occurrence of $A$. The plausibility measure of event $A$ combines the pieces of evidence that enable the occurrence of $A$, that is, avoiding contradiction with $A$. In this situation, the probability of any event $A \subseteq \Omega$, denoted $\operatorname{Pr}(A)$, is imprecise and $\operatorname{Bel}(A)$ and $\operatorname{Pl}(A)$ represent, respectively, the lower and upper probabilities of event $A$, that is, $\operatorname{Pr}(A) \in[\operatorname{Bel}(A), \operatorname{Pl}(A)]$. Two interesting extreme cases of belief and plausibility measures can be obtained by adding constraints to the set of focal elements. When all focal elements are singletons, the belief and plausibility measures become just a single measure: the well-known probability measure $\operatorname{Pr}(A)$. Another special case is encountered when all focal elements are nested (i.e., when they are ordered by a set inclusion), in which case the belief and plausibility measures become, respectively, the necessity and possibility measures. ${ }^{14}$ 


\subsection{Merging Experts' Assessments}

This subsection will reveal how possibility distributions and associated location and uncertainty indicators are able to model the variability of imprecise expert assessments with respect to each criterion. This imprecision encompasses subjectivity and approximation in the utility scale used by experts. Merging imprecise sources of information requires a combination rule. The classical combination rules ${ }^{33}$ presuppose that all sources are reliable (conjunctive mode) or at least one source is reliable (disjunctive mode). The combination used here to build possibility distributions is related to a trade-off mode.

\subsubsection{Methods for Building Possibility Distributions from a Set of Intervals}

Let's consider a set of distinct intervals $\left\{I_{j} \mid j=1, N I\right\}$ as the focal elements and the probability of occurrence of interval $I_{j}$ as the $b b a m\left(I_{j}\right)$ assigned to this interval.

When intervals are nested, that is, $I_{1} \subseteq I_{2} \subseteq \ldots \subseteq I_{N I}$, a possibility distribution $\pi$ may be built from plausibility measure, as proposed in Ref. 34,

$$
\forall \omega \in \Omega, \pi(\omega)=P l(\{\omega\})=\sum_{j=1, N I} m\left(I_{j}\right) \cdot 1_{I_{j}}(\omega) .
$$

When intervals are consistent, that is, $\bigcap_{j=1, N I} I_{j}=I \neq \emptyset$ (where all experts share at least one value), but not nested, two possibility distributions are built. Indeed, let $\forall \omega \in \Omega, \pi_{1}(\omega)=\sum_{j=1, N I} m\left(I_{j}\right) .1_{I_{j}}(\omega)$ and $E_{s}=E_{s-1} \cup E_{\alpha_{s}}\left(\pi_{1}\right)(s=$ $2, r)\left(E_{1}=I\right)$ be the new nested elements built from $\left\{I_{j} \mid j=1, N I\right\}$. In Ref. 34 the $b b a m\left(E_{s}\right)$ are computed as $m\left(E_{s}\right)=\sum_{\left\{I_{j} \text { related to } E_{s}\right\}} m\left(I_{j}\right)$ (each assessment $I_{j}$ being related in a unique way to the smallest $E_{s}$ containing it). Then a possibility distribution $\pi_{2}$ can be defined as $\forall \omega \in \Omega, \pi_{2}(\omega)=$ $\sum_{s=1, r} m\left(E_{s}\right) \cdot 1_{E_{s}}(\omega)$. The possibility distributions $\pi_{1}$ and $\pi_{2}$ are the best possibilistic lower and upper approximations ${ }^{35}$ (in the sense of inclusion) of assessment sets $\left.\left\{I_{j} \mid j=1, N I\right\}\right)$.

In general however, experts' assessment might be neither precise nor consistent. The probability representation and possibility representation correspond, respectively, to extreme and ideal situations; unfortunately, the experts may issue contradictory assessments. This means that the consistency constraint may not be satisfied in practice, that is, $\bigcap_{j=1, N I} I_{j}=\emptyset$. To cope with this situation, the groups of intervals with a nonempty intersection must be built from intervals $\left\{I_{j} \mid j=1, N I\right\}$, which is equivalent to finding subsets $K_{\beta} \subset\{1, \ldots, N I\}, \beta=1, g$ such that: $\bigcap_{j \in K_{\beta}} I_{j} \neq \emptyset$, with $g$ being the number of subsets $K_{\beta}$. These subsets of intervals are called maximal coherent subsets (MCS). This notion was first introduced in Ref. 36, for cases concerning inconsistent rule bases, and then extended to the imprecise probabilistic case. ${ }^{33}$ For each group $K_{\beta}$, two fuzzy subsets $\pi_{1}^{\beta}$ and $\pi_{2}^{\beta}$ are built (as in the previous case when elements are consistent as possibilistic lower and upper approximations of intervals $\left.\left\{I_{j} \mid j \in K_{\beta}\right\}\right)$. 


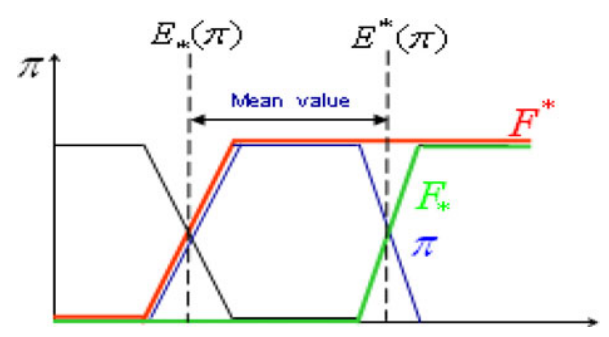

Figure 3. Example of $F^{*}, F_{*}, E^{*}$, and $E_{*}$.

Let possibility distribution $\pi_{1}$ (respectively $\pi_{2}$ ) be the union (denoted $\tilde{U}$ ) of possibility distributions $\pi_{1}^{\beta} \mid \beta=1, g$ (respectively $\pi_{2}^{\beta} \mid \beta=1, g$ ):

$$
\pi_{1}=\tilde{\bigcup}_{\beta=1, g} \pi_{1}^{\beta}\left(\text { respectively } \pi_{2}=\bigcup_{\beta=1, g}^{\tilde{U}} \pi_{2}^{\beta}\right)
$$

then, $\pi_{1}$ and $\pi_{2}$ are the possibilistic lower and upper approximations of intervals $\left\{I_{j} \mid j=1, N I\right\}$. Note that generally, the distributions $\pi_{1}$ and $\pi_{2}$ are multimodal.

By applying the previous methods, the resulting experts' assessment merger is a pair of possibility distributions $\pi_{1}$ and $\pi_{2}$, representing the lower and upper bounds of the plausibility function. Reasoning with the lower distribution (respectively upper distribution) might correspond to a severe risk-aversion position relative to the probability of information (respectively a flexible risk-acceptance position). Consequently, when the ranking of alternatives is the same with both distributions, this implies that the decision is the same regardless of the decisional behavior. In our critical field of application, we have kept both results and only conclude when they corroborate; this avoids any assumption regarding the merging of experts' assessments.

\subsubsection{Definition of Summary Indicators of Possibility Distribution}

Similar to probability distributions, whereby a distribution can be characterized by a limited set of parameters (mean, variance, skewness, etc.), we propose to describe a possibility distribution by the use of meaningful indicators relative to both location and uncertainty. A possibility distribution $\pi$ allows representing some incomplete probability knowledge by bracketing an unknown probability value. We can thus define an upper $\left(F^{*}\right)$ and a lower $\left(F_{*}\right)$ cumulative distribution function, such that $\forall x \in \mathbb{R}, F_{*}(x) \leq F(x) \leq F^{*}(x)$ (Figure 3), with $F^{*}(x)=\Pi([-\infty, x])$ and $F_{*}(x)=N([-\infty, x])$. The difference between $F_{*}(x)$ and $F^{*}(x)$ reflects the uncertainty of the specific information. To quantify this uncertainty, we can use the lower mean value $E_{*}(\pi)=\int_{-\infty}^{+\infty} x d F^{*}(x)$ and the upper mean value $E^{*}(\pi)=$ $\int_{-\infty}^{+\infty} x d F_{*}(x)$. 
Table I. Expressions of the Location and Uncertainty Indicators.

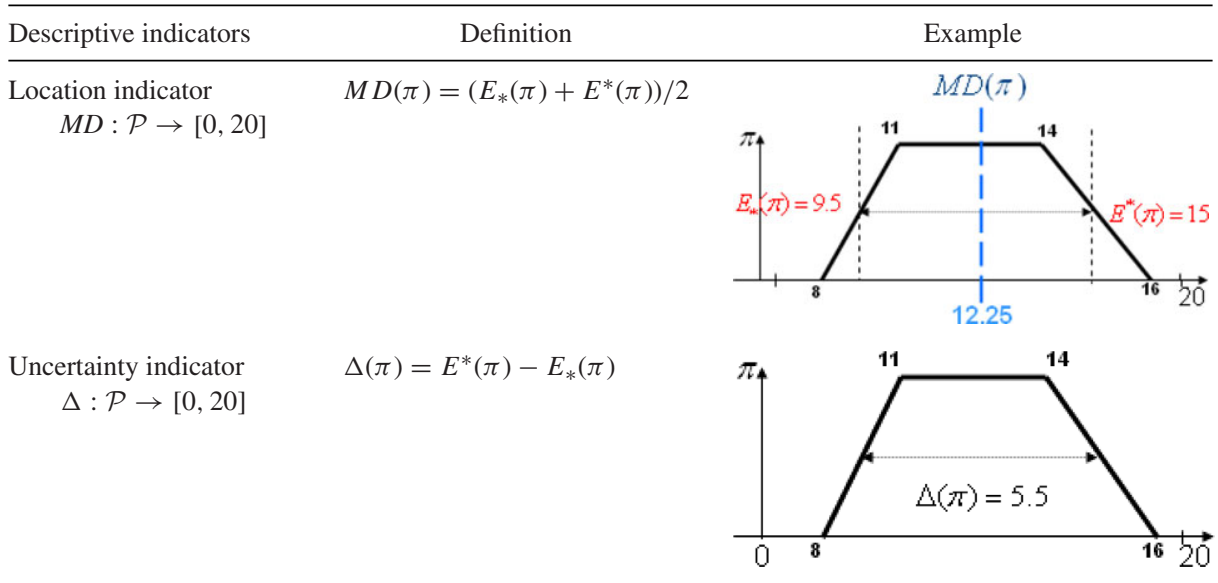

An important property ${ }^{37}$ to be used later is the invariance of values $E_{*}(\pi)$ and $E^{*}(\pi)$ by the linear operations on $\pi$. Since the aggregation operators (i.e., the 2-additive CIs, see Section 3.4) used throughout the paper are linear per the simplex domain, this property will be very useful for the proposed definition of meaningful contribution indicators given in Table I (the support for these indicators is the interval $[0,20]$, which corresponds to the possible values that can be assigned to the degrees of satisfaction in our application). In Table I, $\mathcal{P}$ denotes the set of possibility distributions defined on $[0,20]$.

The expression of $\Delta(\pi)$ allows verifying both extreme cases of uncertainty, that is, the consensus (total precision, $E^{*}(\pi)=E_{*}(\pi)$ ) and total conflict $(\forall \omega \in$ $[0,20], \pi(\omega)=1$; hence $E^{*}(\pi)=20$ and $\left.E_{*}(\pi)=0\right)$. In the consensus case, the uncertainty is $\Delta=0$ and in the total conflict case, it is $\Delta=20$.

Left and right skewness indicators can be defined along the same line ${ }^{29}$ they have not been detailed herein, however, due to their lack of utility in our application context. For multimodal distributions $\pi$, that is, $\pi=\tilde{\bigcup}_{\beta=1, g} \pi^{\beta}$, the preceding approach can be applied separately for each mode, which leads to the following definition of the location indicator:

$$
M D(\pi)=\sum_{\beta=1}^{g} M D\left(\pi^{\beta}\right) / g
$$

For the uncertainty indicator, a similar permode approach can be applied:

$$
\Delta(\pi)=\sum_{\beta=1}^{g} \Delta\left(\pi^{\beta}\right)
$$




\subsection{Aggregation of Experts' Assessments Using the Choquet Integral}

\subsubsection{The Choquet Integral}

The operators of the CI family are interesting due to their inclusion of many compromise operators, which explains their success in a wide array of applications. ${ }^{26}$ Moreover, they can be written in the form of a conventional weighted mean modified by the effects stemming from interactions between criteria. Depending on the application context, only a special case of Choquet fuzzy integrals, known as the 2 -additive $\mathrm{CI},{ }^{38}$ that solely takes into account interactions by pairs will be considered herein. In the following, the notations of the 2-additive CI and its principal properties will be briefly recalled. The expression of the 2-additive Choquet fuzzy integral for an element $\left(x_{1}, \ldots, x_{n}\right) \in \mathbb{R}^{n}$ can be written as follows: ${ }^{39}$ $C I\left(x_{1}, \ldots, x_{n}\right)=\sum_{\mathrm{i}=1}^{\mathrm{n}} v_{\mathrm{i}} x_{\mathrm{i}}-\frac{1}{2} \sum_{i>j} I_{\mathrm{ij}}\left|x_{\mathrm{i}}-x_{\mathrm{j}}\right|$.

This equation involves two types of parameters:

- $v_{i}$ are the Shapley indices, representing the importance of each criterion relative to all others that satisfy $\sum_{i=1}^{n} v_{\mathrm{i}}=1$, which is a natural condition for decision makers;

- the interaction parameters $I_{i j}$ of any pair of performance criteria $(i, j)$ ranging on $[-1 ; 1]$; a positive value means complementarity between the two criteria, a negative value means a redundancy, and a zero value means the criteria are not interacting, that is, preferentially independent; hence, $v_{\mathrm{i}}$ acts as the weights in a common weighted mean.

The $n(n-1) / 2$ parameters of the 2 -additive CI can be identified by the direct expression from the decision makers. Nevertheless, for a higher-quality quantification, more elaborate methods are available. ${ }^{40,41}$ Another important point, directly in line with this paper's contribution, is that the operator $C I$ has a linear form on the simplex domains defined by ranking the evaluations (this ranking allows simplifying the absolute value in the right-hand side of the equation). By considering a permutation on $x_{1}, \ldots, x_{n}$ such that $x_{(1)} \leq \ldots \leq x_{(i)} \leq \ldots \leq x_{(n)}$ (where (.) is a permutation defined on $\{1, \ldots, n\}$ ), the $C I$ can then be written as: ${ }^{42}$ $C I\left(x_{1}, \ldots, x_{n}\right)=\sum_{i=1}^{n} \Delta \mu_{(i)} . x_{(i)}$, where $\Delta \mu_{(n+1)}=0$ and $\Delta \mu_{(i)}=\mu_{(i)}-\mu_{(i+1)}$, with $\Delta \mu_{(i)}=v_{(i)}+\frac{1}{2} \sum_{j>i} I_{(i)(j)}-\frac{1}{2} \sum_{j<i} I_{(i)(j)}$ such that: $v_{i}-\frac{1}{2} \sum_{j \neq i}\left|I_{i j}\right| \geq 0$.

\subsubsection{Aggregation of Possibility Distributions Using the Choquet Integral}

In the general case, the aggregation of possibility distributions by the $\mathrm{CI}$ is based on Zadeh's extension principle. ${ }^{43}$ This principle is very general and can be applied to all distribution shapes, even multimodal. In fact, since we are dealing with piecewise linear distributions and a piecewise linear aggregation operator, then the aggregated possibility distribution is also piecewise linear. Given that the CI assumes a linear form once the evaluation ranking has been determined, the next step entails transposing this property into the possibility distribution space. The ranking of two distributions can actually be set by their intersections: ${ }^{44}$ each time an intersection occurs between the ascending (respectively descending) parts of the distributions, the initial distribution ranking is modified. Each of these intersection points determines, on both sides, the domains $H_{k}$ and $H_{k+1}$, denoted domain $k$ and 

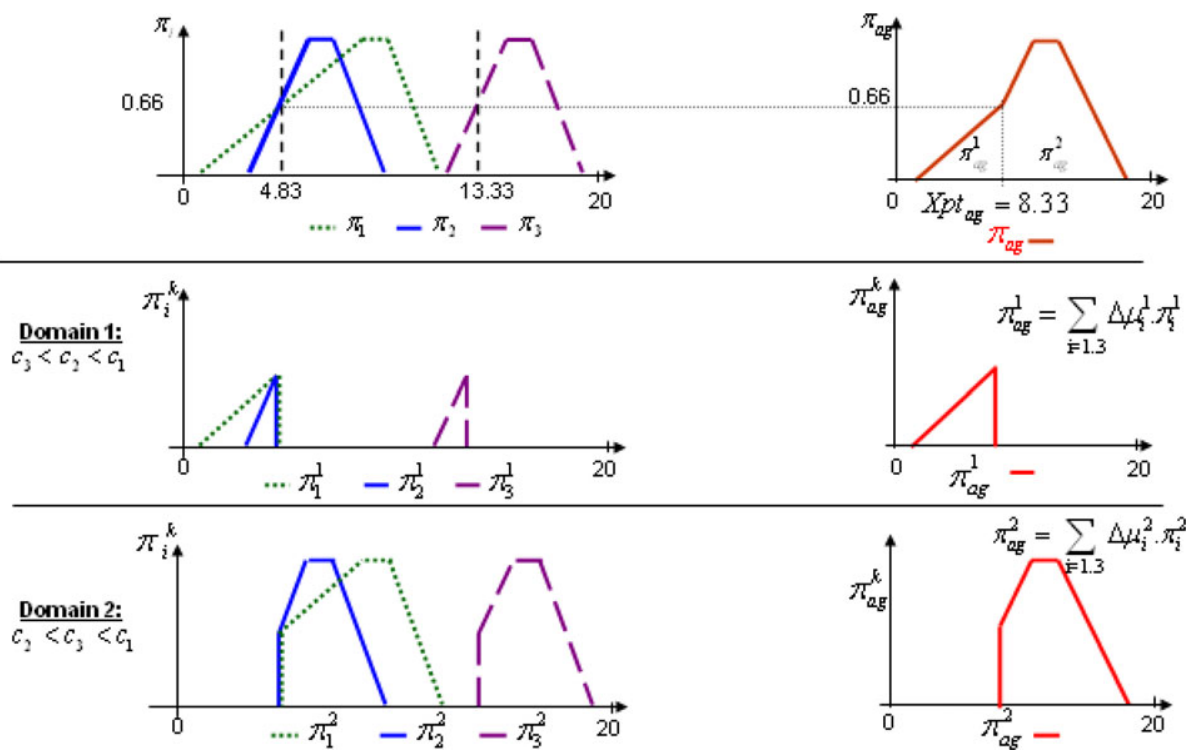

Figure 4. Examples of possibility distribution decomposition by linear domains.

$k+1$ in the remainder of this paper, where the CI offers a linear expression. To perform these computations, each possibility distribution is thus decomposed into well-ranked partial possibility distributions (see an example in Figure 4) and can be subsequently reunified. The aggregated possibility distribution through the CI $\pi_{a g}$ of $n$ possibility distributions $\pi_{1}, \ldots, \pi_{n}$ can indeed be written as the union of elementary aggregated distributions of the $p+1$ disjointed domains defined by the $p$ points of linearity change on the propagated distribution. ${ }^{45}$

$$
\pi_{a g}=\bigcup_{k=1 . . p+1}^{\tilde{U}} \pi_{a g}^{k}=\bigcup_{k=1 . . p+1}^{\tilde{\sum_{i}=1 . . \mathrm{n}}} \Delta \mu_{i}^{k} \cdot \pi_{i}^{k}
$$

where $\pi_{a g}^{k}$ is the partial aggregated distribution relative to the $k^{\text {th }}$ domain, in which the CI has a linear expression, and $\pi_{i}^{k}$ is the partial possibility distribution of the $i$ th possibility distribution involved in the $k^{\text {th }}$ domain ( $\sum$ denotes the sum of possibility distributions).

As an illustration, let's consider the example given in Figure 4. In the left-hand column, the criteria distribution and their decomposition into two linear domains, according to the only ascending intersection point between distributions $\pi_{1}$ and $\pi_{2}$, are plotted. In the right-hand column, the aggregated distribution and its decomposition into the two linear domains are shown.

In the multimodal case, we have observed that the assessments can be represented by lower and upper multimodal distributions for each distribution $\pi_{i}(i=1, \ldots, n)$, with respectively $\pi_{1, i}=\sim \bigcup_{\beta=1, g_{i}} \pi_{1, i}^{\beta}$ and $\pi_{2, i}=\sim_{\beta=1, g_{i}} \pi_{2, i}^{\beta}$. 
Since processing is the same for both lower and upper approximations, let's note $\pi_{i}=\tilde{U}_{\beta=1, g_{i}} \pi_{i}^{\beta}$ in the following computations. The union theorem ${ }^{37}$ states that when multimodal distributions are to be aggregated, the aggregation for each combination of modes is computed and then the $\tilde{U}$ operator is applied. Equation 4 is computed for each combination of modes $\beta=\left(\beta_{1}, \ldots, \beta_{n}\right) \in B$ with $\forall i \in\{1, \ldots, n\}, 1 \leq \beta_{i} \leq g_{i}$ ( $\beta_{i}$ is the index mode of the $i$ th distribution): $\forall \beta \in$ $\mathrm{B}, \pi_{\beta}=\tilde{\bigcup}_{k=1 . . p_{\beta}+1} \pi_{\beta}^{k}=\tilde{\bigcup}_{k=1 . . p_{\beta}+1} \tilde{\sum}_{\mathrm{i}=1 . . \mathrm{n}} \Delta \mu_{i}^{k} \cdot \pi_{i}^{\beta, k}$, which ultimately yields:

$$
\pi_{a g}=\bigcup_{\beta \in \mathrm{B}}^{\sim} \pi_{\beta}
$$

where $p_{\beta}+1$ is the number of domains of linearity relative to mode $\beta$ of $\pi_{a g}$ and $\pi_{i}^{\beta, k}$ is the part of the distribution $\pi_{i}$ within linearity domain $k$ contributing to mode $\beta$ of $\pi_{a g}$.

\subsection{Aggregation of the Uncertainty Indicator using the Choquet Integral}

The indicators associated with a possibility distribution $\pi$ are based essentially on the mean value of the upper and lower probability distribution $E^{*}$ and $E_{*}$. Moreover, we noticed that the CI is linear by domains; the coefficients of the various linear expressions adopted by the CI depend on the ranking of distributions. This statement implies that the aggregation of $E_{*}\left(\pi_{i}\right)$ (respectively $E^{*}\left(\pi_{i}\right)$ ) also depends on such a ranking, which is modified every time an intersection occurs between the ascending (respectively descending) parts of the partial distributions. ${ }^{44}$

Therefore, $E_{*}\left(\pi_{a g}^{k}\right)=\sum_{i=1}^{n} \Delta \mu_{i}^{k} \cdot E_{*}\left(\pi_{i}^{k}\right)$ (respectively $E^{*}\left(\pi_{a g}^{k}\right)=\sum_{i=1}^{n} \Delta \mu_{i}^{k}$. $\left.E^{*}\left(\pi_{i}^{k}\right)\right)$ can only be verified in each domain of linearity, though $E_{*}\left(\pi_{a g}\right)=$ $C I\left(E_{*}\left(\pi_{1}\right), \ldots, E_{*}\left(\pi_{n}\right)\right)$ (respectively $\left.E^{*}\left(\pi_{a g}\right)=C I\left(E^{*}\left(\pi_{1}\right), \ldots, E^{*}\left(\pi_{n}\right)\right)\right)$ does not hold. The following original theorem and corollary have thus been established in Ref. 45:

\section{THEOREM. Union of $E_{*}$ and $E^{*}$.}

Let $\pi_{1}, \ldots, \pi_{n}$ be a piecewise linear distribution and $p$ is the number of points of change of linearity. Let's also denote $\pi$ as one of these distributions. $\pi$ can now be written as $\pi=\tilde{\bigcup}_{k=1 . . p+1} \pi^{k}$, where $\pi^{k}$ are the adjacent linear distributions (not necessarily normalized) ordered from left to right. Let $k_{N}$ be the domain index corresponding to the normalized partial distribution (i.e., the part containing the kernel of $\pi)$. We now have for $k_{N}>1(p=0$ gives a single region $k=1)$ : $E_{*}(\pi)=$ $\sum_{k=1}^{k_{N}} E_{*}\left(\pi^{k}\right)-\sum_{k=1}^{k_{N}-1} E^{*}\left(\pi^{k}\right)$ and $E^{*}(\pi)=\sum_{k=k_{N}}^{p+1} E^{*}\left(\pi^{k}\right)-\sum_{k=k_{N}+1}^{p+1} E_{*}\left(\pi^{k}\right)$.

COROLlary.. Let $\pi$ be a piecewise linear distribution, $\pi=\tilde{\bigcup}_{k=1 . . p+1} \pi^{k}$, where $\pi^{k}$ are the adjacent linear distributions (not necessarily normalized) ordered from left to right and where $p$ is the number of points of change of linearity. We now obtain $\Delta(\pi)=\sum_{k=1}^{p+1} \Delta\left(\pi^{k}\right)$. 
Thanks to the linearity of $C I$ in each domain $k$ and to the invariance of $E_{*}$ and $E^{*}$ by linear transformation, the uncertainty indicator of the aggregated distribution can be written as $\Delta\left(\pi_{a g}^{k}\right)=\sum_{i=1}^{n} \Delta \mu_{i}^{k} \Delta\left(\pi_{i}^{k}\right)$. From the corollary, it can thus be deduced that $\Delta\left(\pi_{a g}\right)=\sum_{k=1}^{p+1} \Delta\left(\pi_{a g}^{k}\right)$, which allows writing

$$
\Delta\left(\pi_{a g}\right)=\sum_{k=1}^{p+1} \sum_{i=1}^{n} \Delta \mu_{i}^{k} \Delta\left(\pi_{i}^{k}\right)
$$

When the aggregated distribution is multimodal ( $g_{a g}$-modal):

$$
\Delta\left(\pi_{a g}\right)=\sum_{\beta=1}^{g_{a g}} \Delta\left(\pi_{a g}^{\beta}\right)
$$

with $\Delta\left(\pi_{a g}^{\beta}\right)=\sum_{k=1}^{p_{\beta}+1} \sum_{i=1}^{n} \Delta \mu_{i}^{\beta, k} \Delta\left(\pi_{i}^{\beta, k}\right)$, where $p_{\beta}+1$ is the number of domains of linearity relative to mode $\beta$ of $\pi_{a g}$, and $\pi_{i}^{\beta, k}$ is the part of the distribution $\pi_{i}$ within linearity domain $k$ contributing to mode $\beta$ of $\pi_{a g}$.

\subsection{Deliberation: Identification of the Criteria to be Discussed First}

During the decision-making deliberation, when experts do not share the same criterion assessment, the head manager must steer the discussion toward the most important criterion for which a mismatch has been observed. A useful tip for the head manager would be to develop a subset of criteria that explain a significant part of the uncertainty in the aggregated possibility distribution. From this perspective, we propose to determine a subset of criteria $I^{*}$ that contributes up to $(100 . \tau) \%$ $(\tau \in[0,1])$ of the uncertainty in the aggregated distribution.

Let $\pi_{1}, \ldots, \pi_{n}$ be the possibility distribution related to each criterion with respect to a given alternative and $\pi_{a g}$ the CI-aggregated distribution. The subset $I^{*}$ is then computed as follows:

$$
I^{*}=\underset{I \in 2^{\{1, ., n\}}}{\operatorname{Arg} \min }\left\{\sum_{i \in I} \Upsilon_{i \Delta} / \sum_{i \in I} \Upsilon_{i \Delta}>\tau . \Delta\left(\pi_{a g}\right)\right\}
$$

with $\Upsilon_{i \Delta}$ being the contribution of the uncertainty indicator $\Delta\left(\pi_{i}\right)$ relative to the distribution $\pi_{i}$, given the uncertainty indicator $\Delta\left(\pi_{a g}\right)$ of the aggregated distribution $\pi_{a g}$. To define $\Upsilon_{i \Delta}$, the approach consists of seeking an additive model of contributions along the lines of the analysis of variance. Our intention therefore is to write 


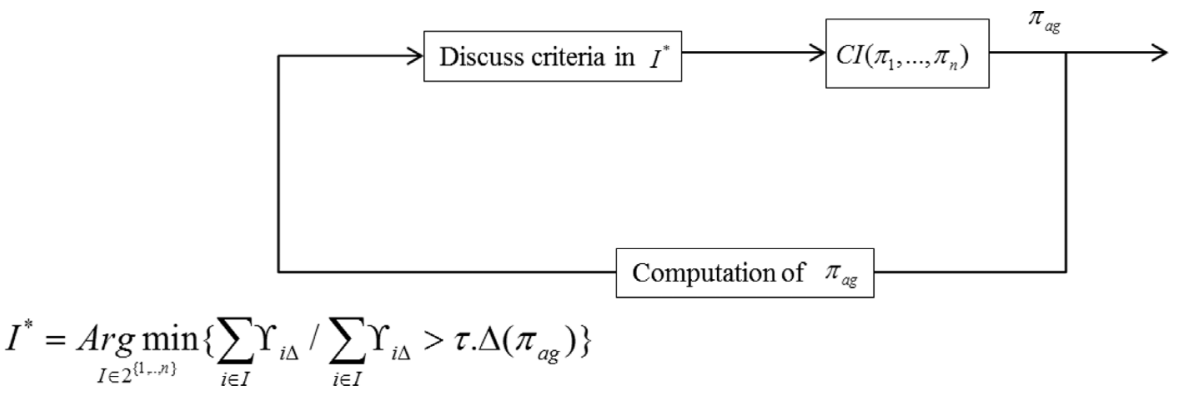

Figure 5. The control loop used to manage the experts' debate.

the uncertainty indicator as:

$$
\Delta\left(\pi_{a g}\right)=\sum_{i=1}^{n} \Upsilon_{i \Delta}
$$

From equation (6), the contribution can thus be written as: $\Upsilon_{i \Delta}=$ $\sum_{k=1}^{p+1} \Delta \mu_{i}^{k} \cdot \Delta\left(\pi_{i}^{k}\right)$

For the multimodal case, the contribution of the uncertainty of each partial evaluation distribution to overall uncertainty is expressed as:

$$
\Delta\left(\pi_{a g}\right)=\sum_{\beta=1}^{g_{a g}} \sum_{i=1}^{n} \Upsilon_{i \Delta}^{\beta}
$$

where $g_{a g}$ is the total number of modes.

The head manager can then use these contributions to recommend that experts focus their attention on the criteria in $I^{*}$ : disagreements on experts' assessments with respect to criteria in $I^{*}$ explain most of the observed uncertainty that disturbs the overall assessment. Experts must verify their respective assessments relative to criteria in $I^{*}$. In the event of a mismatch, either an expert provided an inaccurate or incorrect assessment or else a point of disagreement must obviously be discussed by experts as a priority. In all cases, uncertainties on criteria assessments in $I^{*}$ must be discussed first to facilitate decision making; this may be represented through a control loop (Figure 5) with uncertainty as the control variable.

This control loop actually helps manage the SA-assessment phase when assessments are given by a group of experts. Endsley's SA model is thus produced here by means of a cognitive feedback, whose control variable is the uncertainty in experts' assessments. The notion of comprehension, interpretation, and decision by a group provides an additional complex factor in SA, and an internal control loop of the SA itself (levels 1, 2 and 3) has thus been introduced (Figure 6). Controlling the dynamic of the deliberation phase allows reducing the SA-response time of the group of experts: they are assumed to converge more rapidly on consensual values, if applicable. This approach avoids having to discuss criteria that are points of contention yet with only a minor impact on the final decision. 


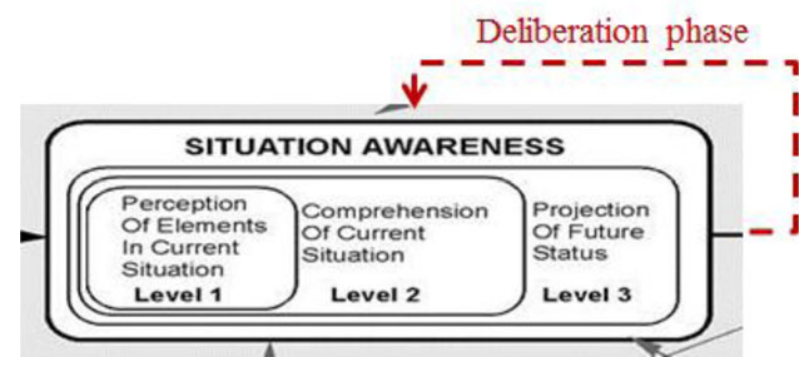

Figure 6. SA and the deliberation phase.

\section{CASE STUDY: DECISION MAKING IN A ROAD TRAFFIC ACCIDENT EMERGENCY MANAGEMENT UNIT}

The selected case study corresponds to a road traffic accident analyzed within the framework of the ANR's (French National Research Agency) ISyCri project (http://w3.univ-tlse1.fr/ceriss/soc/isycri/). This project has focused on emergency situations through two specific perspectives: coordination of the actions of teams involved in the emergency unit (reactivity), and adaptation of this collective action as the situation evolves over time (flexibility). ${ }^{46,47}$ For the present case study, we will consider decision-making issues relative to the group's SA adaptation as the situation changes. The decision-making process is seen as a series of elementary deliberative processes between experts with different cultures yet sharing the same overall objective: resolving emergency issues. Nevertheless, each expert can only refer to his own incomplete point of view corresponding to his area of expertise, information system, mission and goals, rules, and capacities. Sharing in a unique consensual assessment of the situation at any given time is a critical point to make relevant decisions: without a shared assessment of the situation, relevant and consistent coordination of the emergency unit might be missing, and the scheduled implementation of an action plan suitable to the evolving emergency might fail. Controlling the debate of experts involved with the emergency unit is thus a major consideration since such control would avoid wasting time with insignificant points of contention arising during the debate, and instead focuses the discussion on those points of contention exerting significant impact on the final decision.

The scenario considered herein concerns a road traffic accident occurring at a grade crossing. A train crashes into a truck that is transporting a toxic product. Many people are injured and the truck tanker bursts into flames. The tag indicating the product being hauled is illegible after the crash. Fire fighters and police are called to the scene. An emergency unit is created. The area surrounding the grade crossing is cleared and all traffic deviated to facilitate the rescue mission. Fire reaches the truck engine. Fire fighters administer first aid to the injured. The oil tank ignites as well. Emergency medical services are called to evacuate the injured to appropriate medical facilities. The truck tanker appears to be leaking, and a gas is observed to be escaping. The situation becomes much more complex and critical 
within the next few hours. The emergency unit manager, that is, the Prefect of Police in this case, must decide whether "the area is to be completely evacuated" (denoted "Evacuate") versus "sheltering of the population concerned" (denoted "Sheltering"). This decision depends on a number of criteria. First of all, criteria relative to both the physical and psychological injuries sustained must naturally be included. In the situation under consideration, a toxic gas release can also have severe consequences for the environment. It is thus obvious that resolving the emergency situation entails minimizing the number of injuries, psychological distresses, and pollution impacts, yet these objectives cannot be successfully achieved without addressing the relative cost of remedial actions and the way they get reported by the media. Successful closure of the emergency situation also depends, therefore, on the economic cost of response and its coverage in the media.

Finally, five criteria related to the physical and psychological injuries, environmental impacts, and economic and media communication consequences are to be incorporated (note that these may be relevant in most emergency situations). Since this decision carries with it serious consequences, the Prefect of Police, who would be blamed in the event of a disaster, consults a team of experts before making the final decision. Each expert sheds further light on the emergency situation in relation to his dedicated field of expertise and assesses the associated criteria with respect to decision alternatives. An overall collective assessment is broken down into two steps: (i) gathering of assessments for each individual criterion and representation of these assessments by a possibility distribution (i.e., the merging process); and (ii) aggregation of the elementary distributions relative to the criteria to yield an overall evaluation of the alternatives (so-called aggregation process). The decision-making process can then be conducted as follows: (i) the aggregated assessments of alternatives are compared; and (ii) should the preference be insufficiently obvious due to excessive uncertainty, the criteria creating sources of indecision are identified and reviewed in order of priority by the group.

For the considered scenario, five experts are selected by the Prefect of Police to offer their assessments of the five preceding criteria, whose relative importance and interactions are empirically described through the following set of rules:

- The relative importance of "Physical injury consequences" is greater than that of "Psychological consequences" and "Environmental consequences";

- The "Media impact" is more of a decisive factor than "Economic control";

- Managing a crisis without "Physical injury consequences," "Psychological consequences," or "Environmental consequences" cannot be considered a successful situation outcome if both the "Media communication" and "Economic control" fail.

These behavioral rules provide information regarding the relative importance of criteria and the preferential interactions between criteria. These rules have been quantified into a 2-additive CI aggregation model using a Macbeth-like method, ${ }^{48,49}$ capable of transforming qualitative preferences into quantitative ones. ${ }^{41}$

The values of relative importance and criteria interactions are shown in Figure 7, which corresponds to screen captures from the DSS developed during the $I S y C r i$ project. The Shapley indices relative to each criterion (i.e., physical injury consequences, psychological consequences, environmental consequences, media 


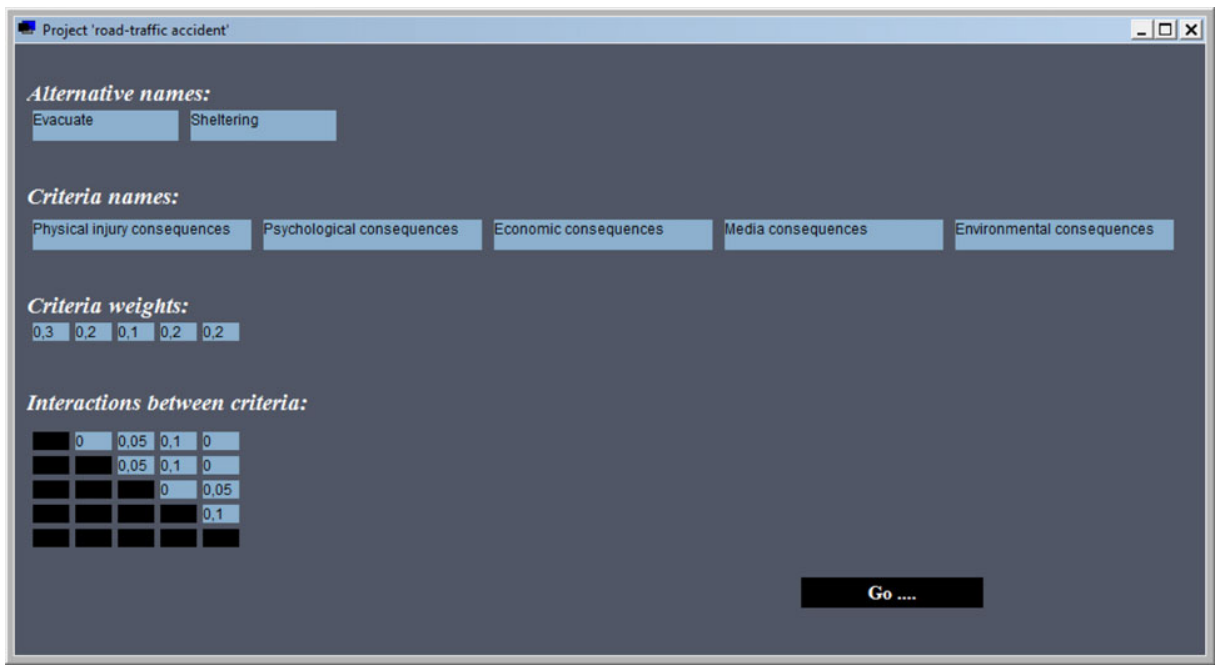

Figure 7. Man-machine interface parameters.

consequences, and economic consequences) equal respectively: 0.3, 0.2, 0.2, 0.2, and 0.1 . The interaction values are: $I_{\text {economic, physicalinjury }}=0.05, I_{\text {economic, } \text { psychological }}=$ $0.05, I_{\text {media, physicalinjury }}=0.1, I_{\text {media, } \text { psychological }}=0.1, I_{\text {media, environmental }}=0.1$, and $I_{\text {economic,environmental }}=0.05$. As an example, the Shapley index relative to "Physical injury consequences" is greater than both the "Psychological consequences" and "Environmental consequences" index values; the interaction coefficients of all three primary criteria (i.e., physical injury consequences, psychological consequences, and environmental consequences) with the secondary criteria (media consequences and economic consequences) are non-negligible. The Prefect of Police can then either validate or just slightly modify these values through application of the man-machine interface proposed in Figure 7.

Figure 8 presents the Smith's expert's assessment of "Physical injury consequences" relative to the alternative "Evacuate."Expert Smith believes that evacuating the entire accident area would monopolize all the patrol cars, fire engines, and ambulances and moreover block the main roads, which could prove detrimental to the evacuation of injured persons. To enter his opinion, the expert moves the cursors from "Yes" to "No": the closer to "Yes" (respectively "No"), the more satisfactory (respectively unsatisfactory) the alternative with respect to the given criterion. "Yes" (respectively "No") reflects complete satisfaction (respectively dissatisfaction) with the given criterion. In this example, Smith is not completely opposed to the alternative "Evacuate" yet still needs to be convinced. All of the experts' assessments have been synthesized in Table II. The intervals placed on a [0, 20] scale in Table II represent imprecise assessments provided by experts through the man-machine interface displayed in Figure 8: the locations of the cursors indicate the lower and upper bounds of the interval representing the expert's imprecise assessment (see the corresponding imprecise assessment in the right-hand corner in Figure 8). In 


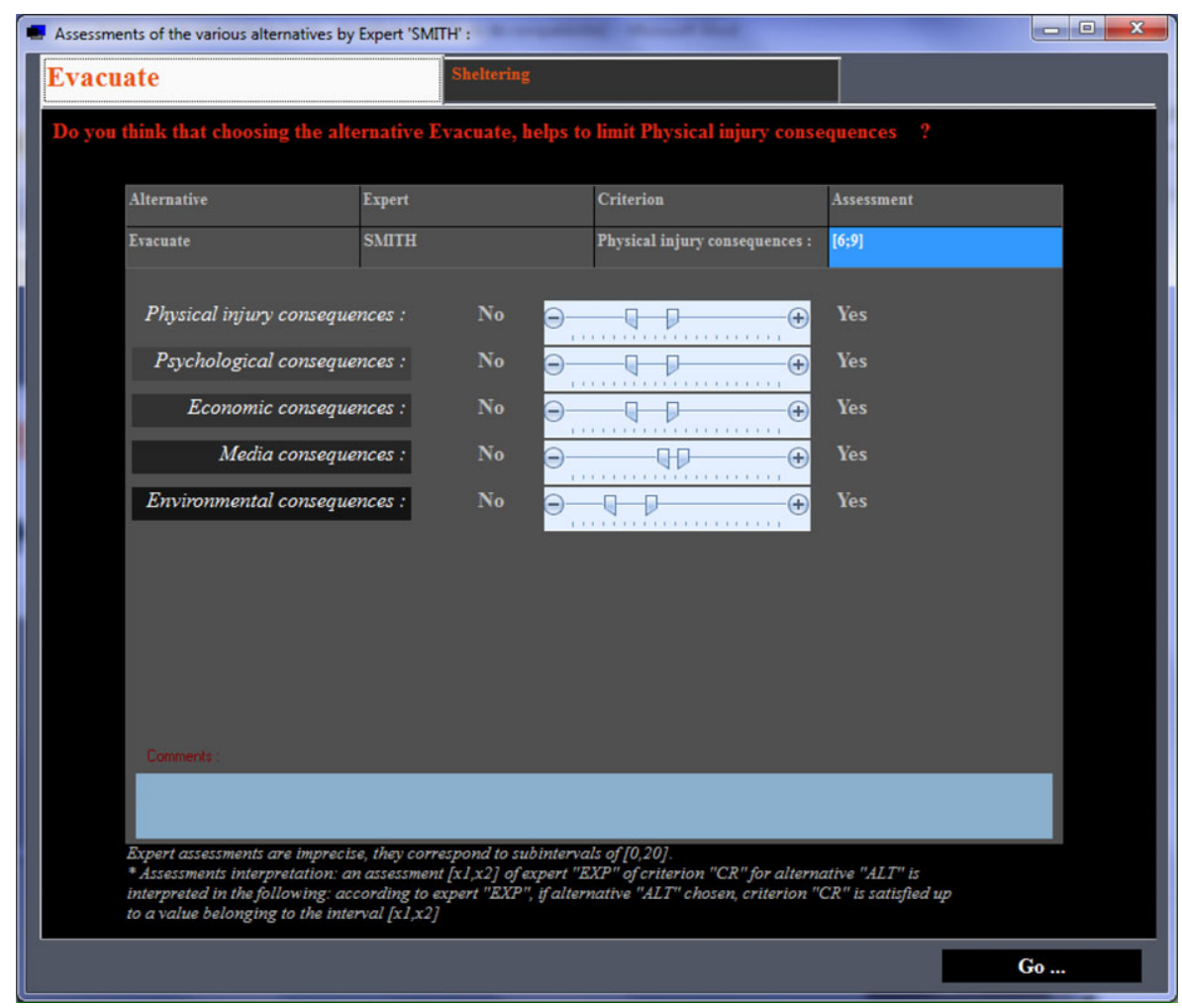

Figure 8. Assessments of the various alternatives by expert Smith.

Table II. Presentation of Each Expert's Assessments.

\begin{tabular}{|c|c|c|c|c|c|c|c|c|c|c|}
\hline \multicolumn{11}{|c|}{ Expert assessments: Consequences of criteria } \\
\hline & \multicolumn{2}{|c|}{ Physical injury } & \multicolumn{2}{|c|}{ Psychological } & \multicolumn{2}{|c|}{ Economic } & \multicolumn{2}{|c|}{ Media } & \multicolumn{2}{|c|}{ Environmental } \\
\hline & Evacuate & Sheltering & Evacuate & Sheltering & Evacuate & Sheltering & Evacuate & Sheltering & Evacuate & Sheltering \\
\hline $\begin{array}{c}\text { Expert 1: } \\
\text { Smith }\end{array}$ & {$[6,9]$} & {$[10,13]$} & {$[6,9]$} & {$[13,16]$} & {$[6,9]$} & {$[15,17]$} & {$[9,10]$} & {$[8,11]$} & {$[4,7]$} & {$[15,17]$} \\
\hline $\begin{array}{l}\text { Expert 2: } \\
\quad \text { Demoran }\end{array}$ & {$[6,9]$} & {$[12,15]$} & {$[7,10]$} & {$[13,16]$} & {$[6,9]$} & {$[15,17]$} & {$[9,10]$} & {$[8,11]$} & {$[4,7]$} & {$[15,17]$} \\
\hline $\begin{array}{l}\text { Expert 3: } \\
\text { Agrelle }\end{array}$ & {$[5,9]$} & {$[12,15]$} & {$[6,9]$} & {$[15,17]$} & {$[6,9]$} & {$[15,17]$} & {$[9,10]$} & {$[8,11]$} & {$[8,11]$} & {$[13,16]$} \\
\hline $\begin{array}{r}\text { Expert 4: } \\
\text { Zattief }\end{array}$ & {$[4,8]$} & {$[12,15]$} & {$[6,9]$} & {$[15,16]$} & {$[4,7]$} & {$[15,17]$} & {$[8,11]$} & {$[7,10]$} & {$[4,7]$} & {$[13,16]$} \\
\hline $\begin{array}{c}\text { Expert 5: } \\
\text { Kusto }\end{array}$ & {$[4,7]$} & {$[4,7]$} & {$[7,9]$} & {$[4,7]$} & {$[4,7]$} & {$[15,17]$} & {$[8,11]$} & {$[7,10]$} & {$[8,11]$} & {$[13,16]$} \\
\hline
\end{tabular}

this case study, each expert is considered as a multispecialist and then authorized to issue an opinion for each criterion.

Figure 9 presents the results of this merging process with respect to each criterion, derived from the assessments listed in each column of Table II. Each image in Figure 9 displays the approximate upper and lower possibility distributions (as 


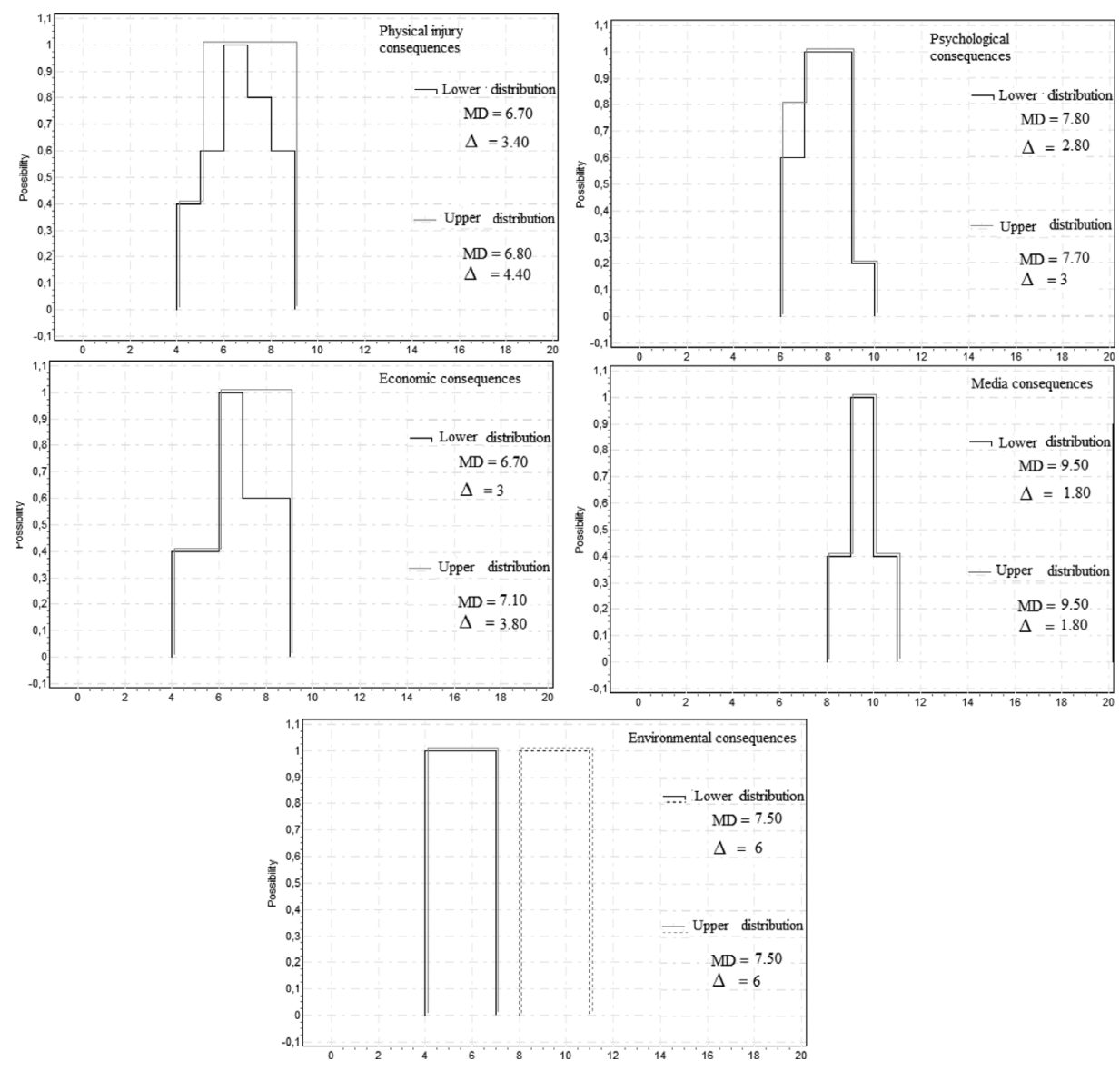

Figure 9. Results of the merging process for the "Evacuate" alternative.

introduced in Section 3) for the set of intervals in Table II. The results of this merging process for all criteria illustrate the dispersion in experts' assessments (as observed by the uncertainty indicator) relative to the criteria for alternative "Evacuate". Nevertheless, the assessments remain consistent (i.e., unimodal relative distributions) except for the "Environmental consequences" criterion, which shows divergence (bimodal distribution). For four of the five criteria, imprecise assessments of the "Evacuate" alternative are consistent yet not nested (e.g., for the "Physical injury consequences" criterion, $[4 ; 8] \cap[5 ; 9] \neq \emptyset$, but $[4 ; 8] \not \subset[5 ; 9]$ and $[5 ; 9] \not \subset[4 ; 8])$; hence, a set of nested intervals, $[6 ; 7] \subset[6 ; 8] \subset[5 ; 9] \subset[4 ; 9]$, is first associated with them, as explained in Section 3.3. The distributions can then be built for the "Physical injury consequences," "Psychological consequences," "Economic consequences," and "Media consequences" criteria. As for the "Environmental consequences" criterion, two opposing assessments (poor vs. ambivalent assessments) are clearly apparent, which explains the resulting bimodal possibility distributions 
for this criterion. The MCS search allows identifying the two modes first; then, the possibility distributions can be computed.

The upper and lower distributions feature the same level of support (i.e., assessments with a nonzero degree of possibility) in our example (Figure 9). However, the set of completely possible assessments (i.e., distribution kernel) for the upper and lower possibility distributions may be distinct: the kernels of upper and lower distributions relative to "Physical injury consequences," "Psychological consequences," and "Economic consequences" criteria significantly differ. Such is not the case for "Media consequences" and "Environmental consequences" criteria, for which all the MCS are nested. This result suggests that the decision might change depending on the decisional behavior of the decision maker. As previously stated, reasoning using the lower distribution (respectively upper distribution) could correspond to a severe risk aversion position regarding the probability of information (respectively to a flexible risk acceptance position). In our critical field of application, we retain both these results and only announce a conclusion when they corroborate. A decision is only made once the ranking of alternatives with both distributions is identical; in other words, a unique robust conclusion can be drawn regardless of the decisional behavior.

Disparities are also observed relative to criteria for the "Sheltering" alternative (see Table II and Figure 10).

Furthermore, the collated assessments present two clearly distinct points of view for the "Psychological consequences" and "Physical injury consequences" criteria (from divergences modeled by bimodal distributions). While the expert Kusto believes that "Sheltering" would have detrimental impacts from the point of view of both the "Physical injury consequences" and "Psychological consequences" criteria, the other experts consider "Sheltering" as a rather satisfactory solution with respect to these criteria. This outcome explains the observed inconsistencies for these two criteria in the distributions stemming from the merging process (Figure 10). Regardless of the selected possibility distribution (i.e., upper vs. lower bound), it is in fact bimodal: the first distribution mode corresponds to expert Kusto's point of view, and the second mode to the merging of all other experts' assessments. Let's note that for the second mode, the kernels of the upper and lower distributions differ: the completely possible values depend on the selected outcome of the merging process, that is, on the decisional attitude regarding error risk. The decision might then differ depending on the decisional behavior (level of risk attitude) of the Prefect of Police. Once again, instead of choosing a particular decisional behavior, both computations are retained in our approach, and a decision is made if and only if both upper and lower distributions yield the same conclusions (same ranking of alternatives).

Once this merging process has been applied to all criteria and both alternatives, the aggregation step is then executed. The five criteria distributions resulting from the merging process can now be aggregated: the outcome of this aggregation process will facilitate the comparison of competing alternatives for the Prefect. Computations are processed for both the upper and lower approximations. The aggregation of criteria distributions is processed using the 2-additive CI depicted in Figure 7. Figure 11 provides the outcome of the aggregation process for the "Evacuate" alternative, while Figure 12 shows the outcome for the "Sheltering" alternative. 


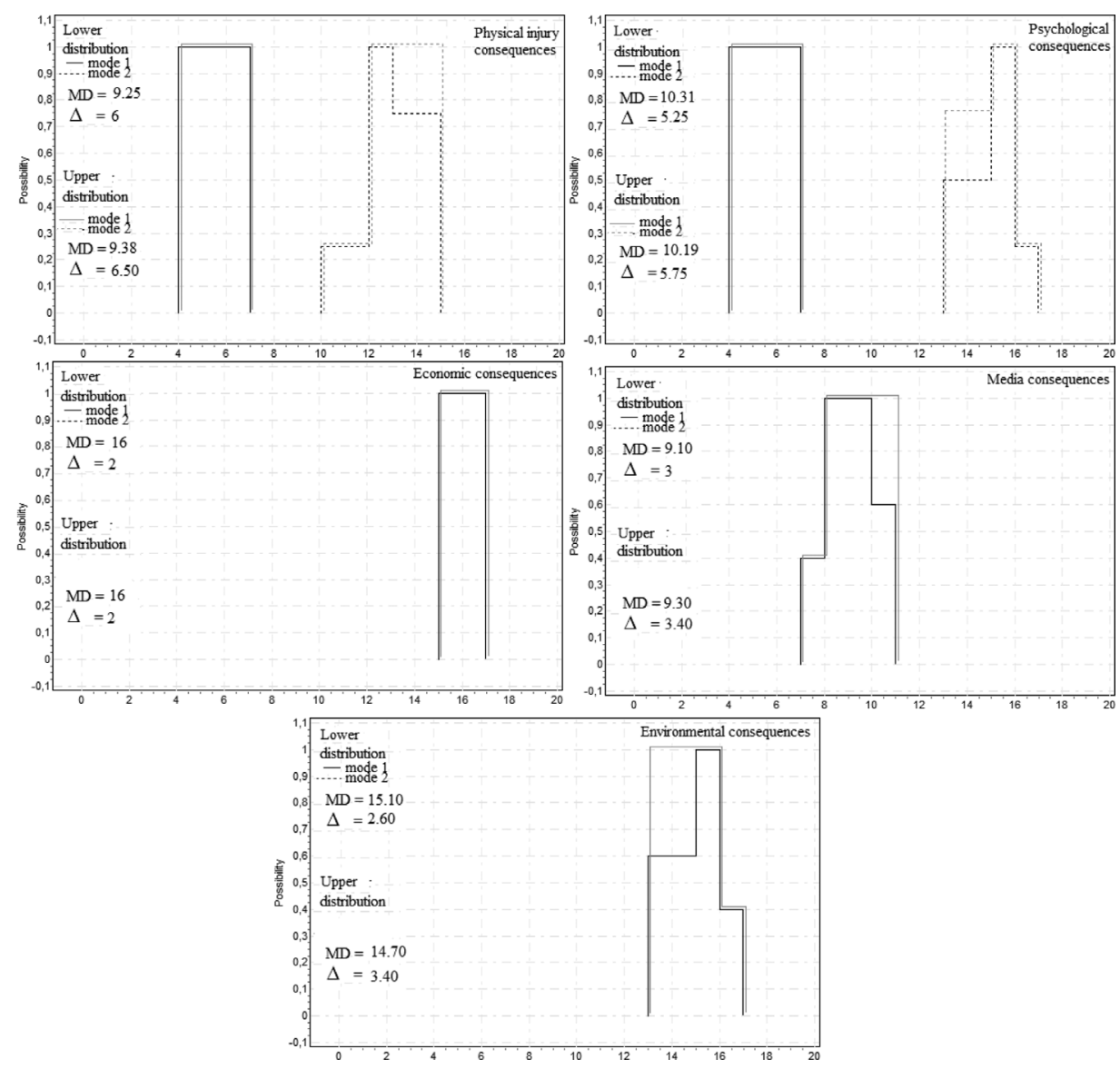

Figure 10. Results of the merging process for the "Sheltering" alternative.

For the "Evacuate" alternative, this aggregation process nearly provides the same result for the location indicator (7.26 vs. 7.25) regardless of the upper or lower outcome model selected for the merging process, that is, regardless of decisional behavior. In contrast, the uncertainty indicator (4.12 vs. 4.65$)$ is distinct and depends on the decisional behavior. Distributions are accompanied by their aggregation process indicator values shown on the DSS screen shots.

For the "Sheltering" alternative, this aggregation process produces a bimodal distribution for the lower approximation and a unimodal distribution for the upper approximation. Note that two criteria ("Physical injury consequences" and "Psychological consequences") are bimodal in the case of "Sheltering". Consequently, we can theoretically imagine that the aggregated distribution in Figure 12 should contain four modes; however, the $\tilde{U}$ operator prevents this proliferation of modes whenever the union theorem (see Equation 5) is applied (see Figure 13 for the lower distributions of both alternatives). 


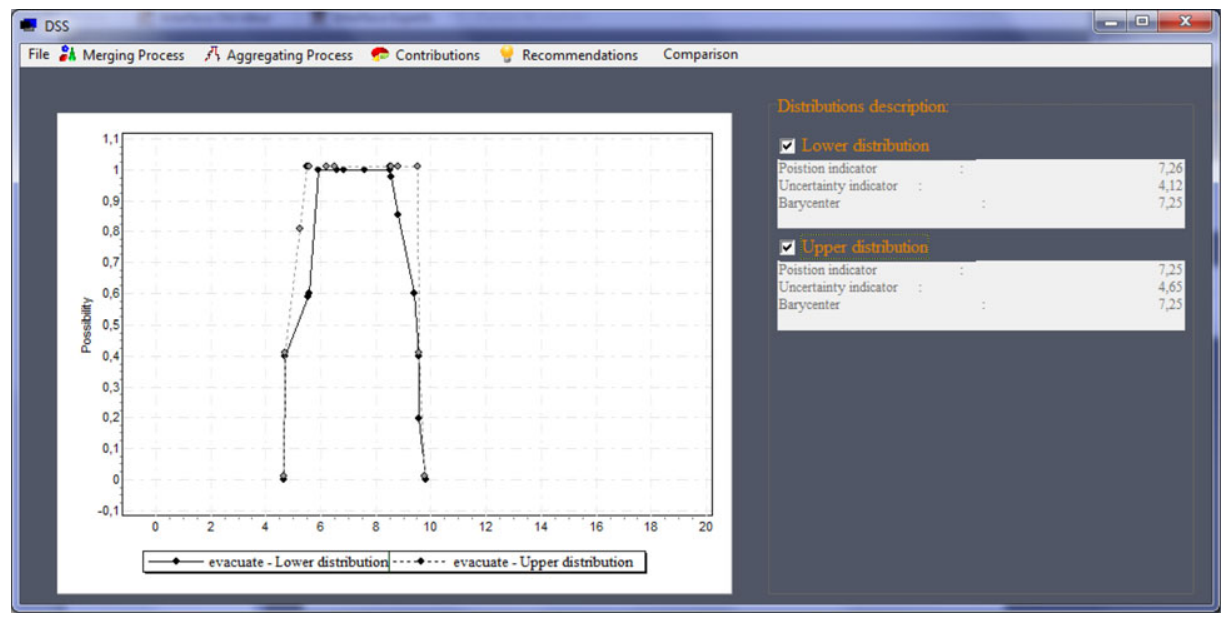

Figure 11. Outcome of the aggregation process-"Evacuate" alternative.

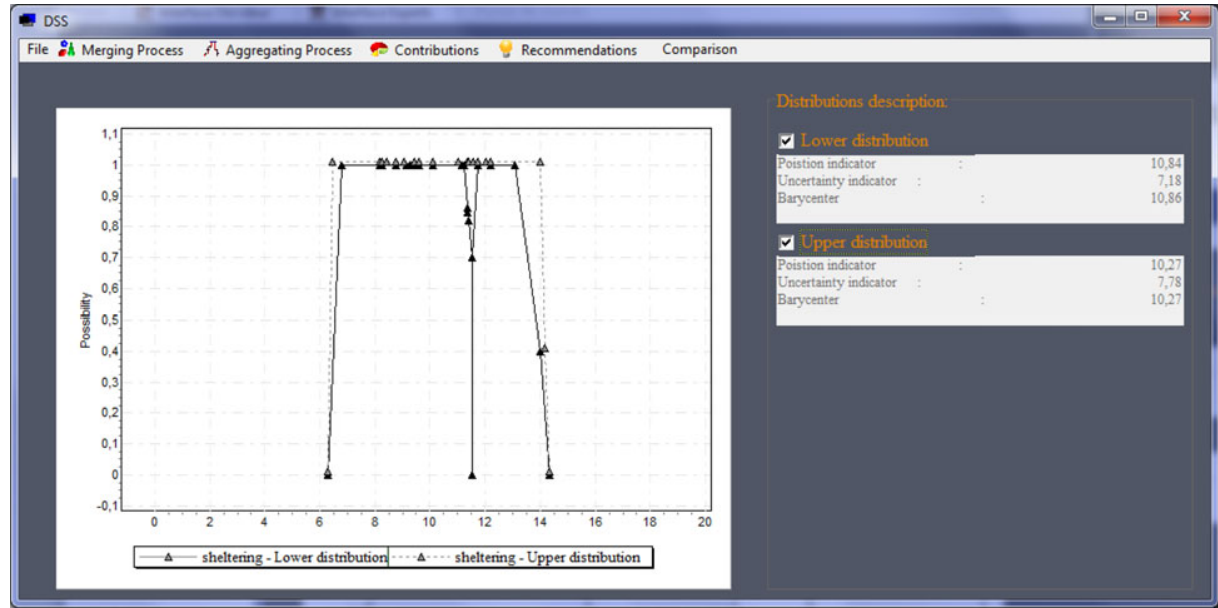

Figure 12. Outcome of the aggregation process—“Sheltering" alternative.

Evacuate

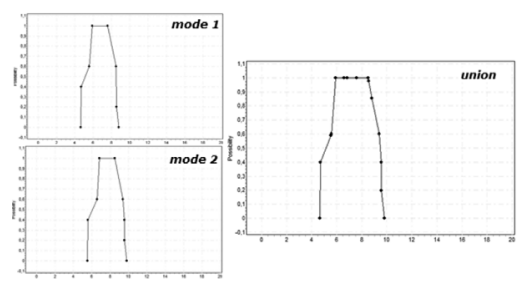

Sheltering

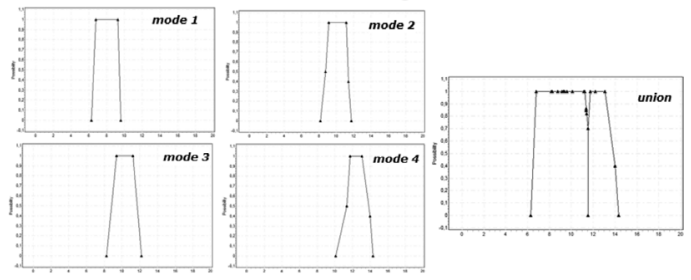

Figure 13. Modes and union of distributions (lower distributions). 
In our example, the support for the aggregated distributions of the "Evacuate" alternative equals [4.65, 9.82] for both the upper and lower distributions. The kernels are [5.92, 8.5] for the lower distribution and [5.5, 9.5] for the upper. For the "Sheltering" alternative, the support is $[6.3,14.35]$ and the kernel $[6.45,14]$ for the upper distribution, and since the lower distribution is bimodal, two kernels are referenced $([6.8,11.22]$ and $[11.72,13.1])$.

Alternatives $A l^{q}$ can now be compared. A Pareto order could, as an example, be introduced. Let $M D\left(\pi^{q}\right)$ be the position indicator and $\Delta\left(\pi^{q}\right)$ the uncertainty indicator of $A l^{q}$, then $A l^{q}$ is the preferred alternative if it is not dominated in the sense of the following Pareto order:

$$
\begin{aligned}
& \left(M D\left(\pi^{q}\right), \Delta\left(\pi^{q}\right)\right) \prec \text { Pareto }\left(M D\left(\pi^{q^{\prime}}\right), \Delta\left(\pi^{q^{\prime}}\right)\right) \Leftrightarrow \\
& \left(M D\left(\pi^{q}\right)+\Delta\left(\pi^{q}\right)<M D\left(\pi^{q^{\prime}}\right)\right) \text { or }\left(M D\left(\pi^{q}\right)<M D\left(\pi^{q^{\prime}}\right) \text { and } \Delta\left(\pi^{q^{\prime}}\right)\right. \\
& \left.\quad \leq \Delta\left(\pi^{q}\right)\right)
\end{aligned}
$$

This expression means that an alternative $A l^{q}$ with a higher location indicator than alternative $A l^{q \prime}$ is a better choice than $A l^{q \prime}$ if its uncertainty is lower or if the location indicator difference is significant (other more flexible orders may of course be specified).

A comparison of two alternatives begins with the computation of the Pareto ranking. If one alternative is preferred to the other in the sense of the Pareto order, then the comparison ends. Otherwise, this means that uncertainty is too strong and likely affecting the decisional process to draw any conclusion. Further analysis must be carried out: the criteria that most significantly contribute to this uncertainty must be identified before the discussion can resume.

Moreover, let's now consider the lower distribution model for the merging process: the following can then be computed (with index 1 for "Evacuate" and 2 for "Sheltering"):

$$
\left(M D\left(\pi^{A l^{1}}\right), \Delta\left(\pi^{A l^{1}}\right)\right)=(7.26,4.12) \text { and }\left(M D\left(\pi^{A l^{2}}\right), \Delta\left(\pi^{A l^{2}}\right)\right)=(10.84,7 .
$$

18). This implies that: $\left(M D\left(\pi^{A l^{1}}\right), \Delta\left(\pi^{A l^{1}}\right)\right) \nprec_{\text {Pareto }}\left(M D\left(\pi^{A l^{2}}\right), \Delta\left(\pi^{A l^{2}}\right)\right)$ and:

$$
\left(M D\left(\pi^{A l^{2}}\right), \Delta\left(\pi^{A l^{2}}\right)\right) \nprec_{\text {Pareto }}\left(M D\left(\pi^{A l^{1}}\right), \Delta\left(\pi^{A l^{1}}\right)\right) .
$$

Neither of the alternatives dominates the other in the Pareto order sense since $7.26+4.12=11.38>10.84$ and $M D\left(\pi^{A l^{1}}\right)=7.26<M D\left(\pi^{A l^{2}}\right)=10.84$; however, $\left.\Delta\left(\pi^{A l^{2}}\right)>\Delta\left(\pi^{A l^{1}}\right)\right]$. An analysis of the upper distribution provides the same conclusion. Hence, the Prefect cannot make a decision: the preference for "Sheltering" falls short of being tangible enough. He must have his experts review their arguments to verify whether these discrepancies in the assessments are justifiable.

The uncertainty indicator is in fact quite high for both alternatives, especially "Sheltering". This uncertainty is due to the uneven assessments of experts relative to the same criterion. The quantitative analysis of this uncertainty requires computing the contribution of each criterion to the aggregated distribution uncertainty, that is, as modeled by the uncertainty indicator (see Equations 9 and 10). Figure 14 shows that 


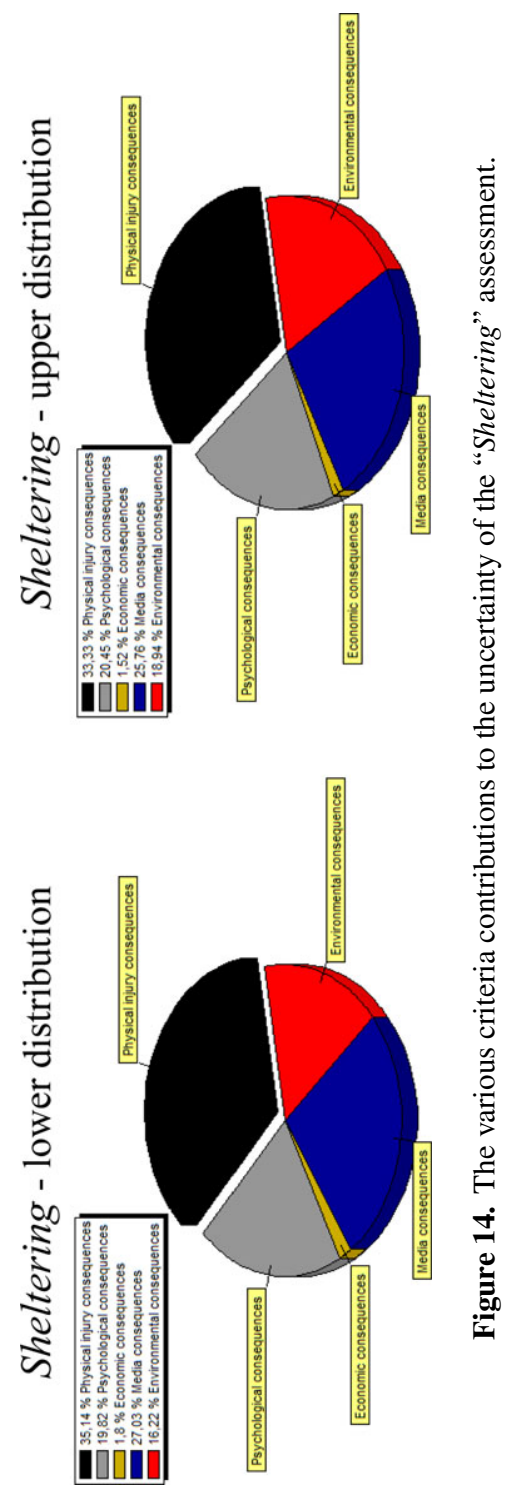


the "Physical injury consequences" criterion contributes most significantly to the aggregated distribution uncertainty for the "Sheltering" alternative, regardless of the decisional attitude (i.e., regardless of the approximate distribution). The "Physical injury consequences" criterion explains more than $30 \%$ of total uncertainty on its own: both the uncertainty and relative importance of this criterion are significant (these conclusions are the same for both the upper and lower distributions). This "Physical injury consequences" uncertainty is what mainly prevents the Prefect from making his decision. Hence, "Physical injury consequences" constitutes a point of contention with a significant impact on the final decision and, as such, must be reviewed as a priority by the group of experts.

From this analysis of criteria contributions to the uncertainty of the aggregated distribution, it can be determined that a significant portion of uncertainty pertains to the "Physical injury consequences" criterion for the "Sheltering" alternative, regardless of the approximation distributions. For the "Sheltering" alternative, this specific criterion will then be the one raised again in discussion as a priority by experts. The aim here is to explain the causes of these detected divergences (observation, interpretation, understanding, school of thought, professional liability, etc.). The SA debate concerning expert assessments focuses on uncertainty as a control variable to distinguish the major controversial and decisional factors simultaneously. In this example, uncertainty of the "Physical injury consequences" criterion mainly disturbs the Prefect in reliably comparing the two competing alternatives presented at the time. The Prefect must ask experts to review their assessments with respect to this criterion, which contributes most to his state of indecision. Four experts share consistent assessments (see Table II), but Kusto disagrees with them relative to this same criterion for the "Sheltering" alternative. Let's now imagine that this isolated expert Kusto is convinced by the remaining group of four experts during the controlled deliberation, offering a revised assessment $[10,13]$ for this "Physical injury consequences" criterion (see Figure 15, left-hand side).

The new distribution for the "Physical injury consequences" criterion stems from the merging process and is now presented in Figure 15 (right-hand side). This

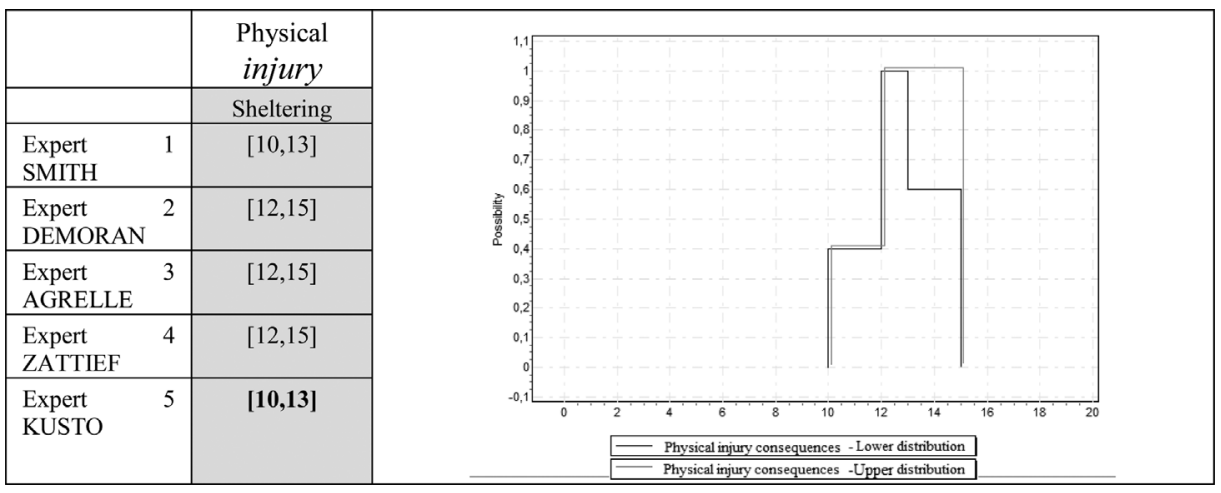

Figure 15. Expert Kusto changes his mind regarding the "Physical injury consequences" criterion-The merging process resumes. 


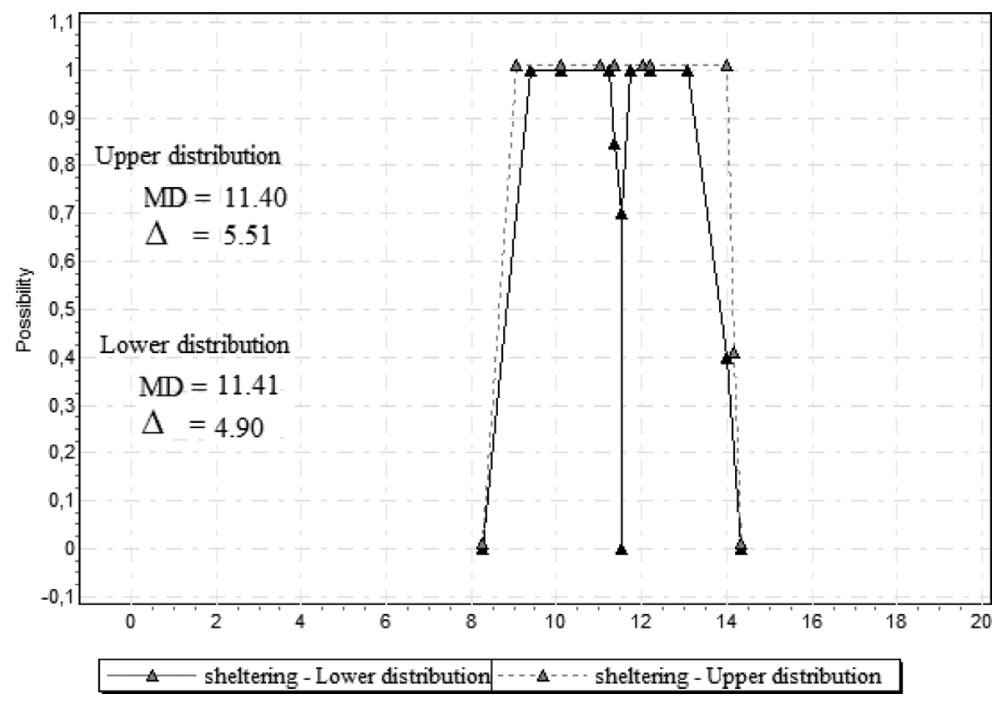

Figure 16. Aggregated "Sheltering" distribution after Expert Kusto has changed his mind.

new distribution is no longer multimodal; some uncertainty remains, yet critical divergence is no longer apparent.

From these new results, Figure 16 shows the new aggregated distributions (from the upper and lower merging approximations) for the "Sheltering" alternative.

Uncertainty in the overall assessment of the "Sheltering" alternative has significantly decreased because of Kusto's revision regarding "Physical injury consequences": 4.90 (respectively 5.51) for the lower (respectively upper) distribution versus greater than 7.18 (respectively 7.78). From the standpoint of the lower distribution, it can be verified that: $7.26+4.12=11.38<11.41$; hence, "Sheltering" is preferred to "Evacuate" in the sense of the Pareto order. From the standpoint of the upper distribution, neither alternative dominates the other: $(7.25+4.65=11.911 .4$ and 5.514.65). As mentioned above, a robust decision is made if and only if both the upper and lower distributions yield the same conclusion. Such is still not the case herein. Although the vagueness of the decision has been significantly reduced, the comparison does not yet allow the Prefect of Police to conclude on a choice.

For the "Sheltering" alternative, besides the "Physical injury consequences" criterion, it can be considered that the "Media consequences" (at 27.3\%) (respectively $25.8 \%$ ) and "Psychological consequences" (19.8\%) (respectively 20.5\%) criteria also significantly contribute to the uncertainty of the lower (respectively upper) aggregated distribution.

The experts do not completely agree regarding the media's reaction as to whether the "Sheltering" alternative is to be selected. Some experts believe this could be a mistake from the point of view of the precautionary principle, while others think that when the evacuation order will be announced and reinforced by media messaging, the risk is run of causing panic, which would complicate the 
crisis-management task. Their assessments however remain close to one another (Table II). Uncertainty with the lower (respectively upper) distribution equals $\Delta=3$ (respectively $\Delta=3.4$ ) relative to the "Media consequences" criterion, which simply means that the strong contribution of "Media consequences" to overall uncertainty is mainly due to the high "weight" ascribed to the criterion through equations (see Equations 9 and 10) in this assessment context. In other words, this criterion is of such high importance herein that even a slight divergence could induce severe impacts on the decision. The Prefect however is unable to influence the "Media consequences" criterion since his experts already share roughly the same qualitative opinion regarding this criterion.

In contrast, uncertainty of the "Psychological consequences" criterion is more significant, and it may prove useful for experts to reconsider this point to validate their opinions. Thanks to the DSS, the Prefect now knows which points must first be redeliberated by experts: assessments of those criteria with high relative importance and a significant uncertainty contribution. Once again, Kusto appears to be isolated and disagrees with the other experts regarding the "Psychological consequences" criterion; he is once again convinced by the group of four and issues a new assessment $[13,16]$ for this criterion (see Figure 17, left-hand side).

The new distribution for the "Psychological consequences" criterion stems from the merging process and is shown in Figure 17 (right-hand side). The revised upper and lower distributions are no longer multimodal.

From these updated results, Figure 18 displays the new aggregated distributions (from lower and upper merger approximations) of the "Sheltering" alternative.

At this point, the "Sheltering" assessments no longer contain divergences (i.e., no more bimodal distributions). It can then be verified that the "Sheltering" alternative is now preferable to the "Evacuate" alternative in the sense of the Pareto order for both lower and upper distributions $(7.26+4.12=11.38<12.35$ and $7.25+4.65=11.9<12.43)$. In this case, the conclusion now becomes obvious (Figure 19).

The reduction in assessment uncertainty has allowed concluding in three iterative steps. Thanks to the DSS, the Prefect of Police has managed the deliberation process to reduce uncertainties in the experts' assessments as quickly as possible. He has indeed been able to identify the criteria that prevented him from making a reliable decision to focus the debate on critical points of contention. The experts then rapidly reached agreement and the Prefect could finally make his decision. The proposed approach allows orienting the debate to efficiently converge on consensual SA whenever possible. This appears as a means of controlling the deliberation phase, which is intended to efficiently reduce the debate duration (a critical aspect of emergency management) by focusing the discussion on the points of contention exerting the greatest impact on the final decision.

\section{CONCLUSIONS}

The issues considered in this paper relate to the decision-aiding functionalities in the context of an emergency management unit composed of experts providing imprecise assessments on multiple criteria that are to be taken into account to yield a 


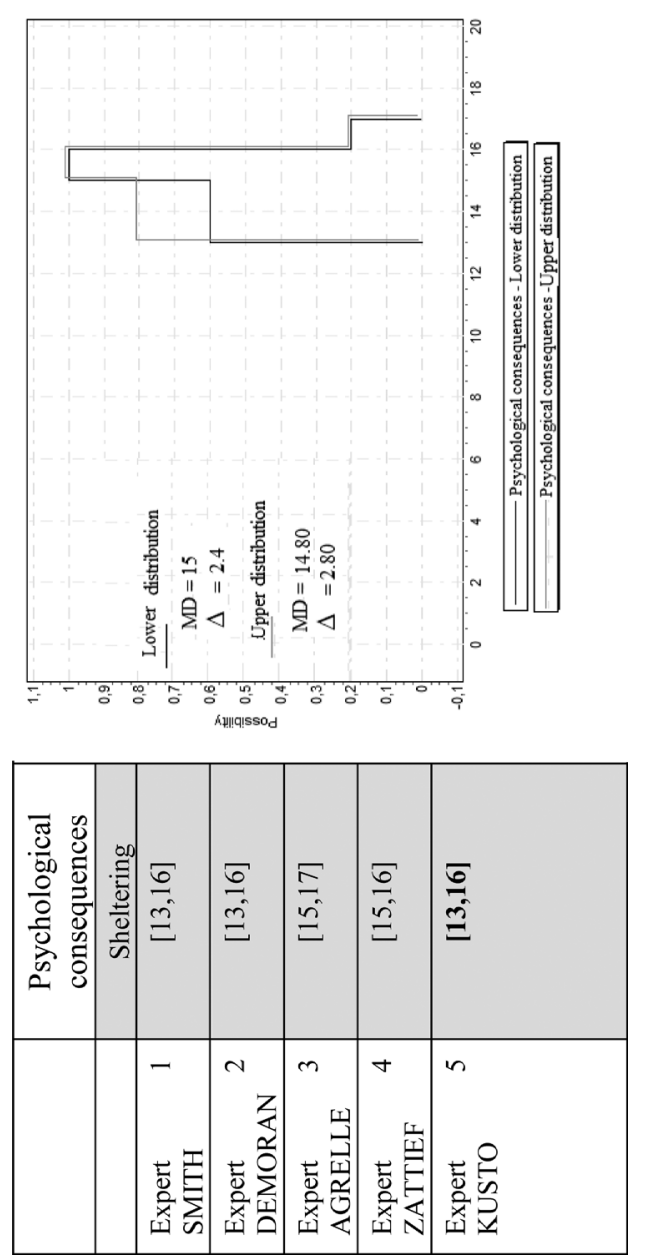

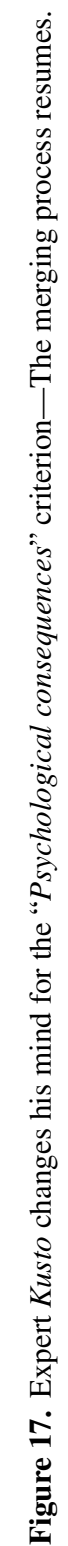




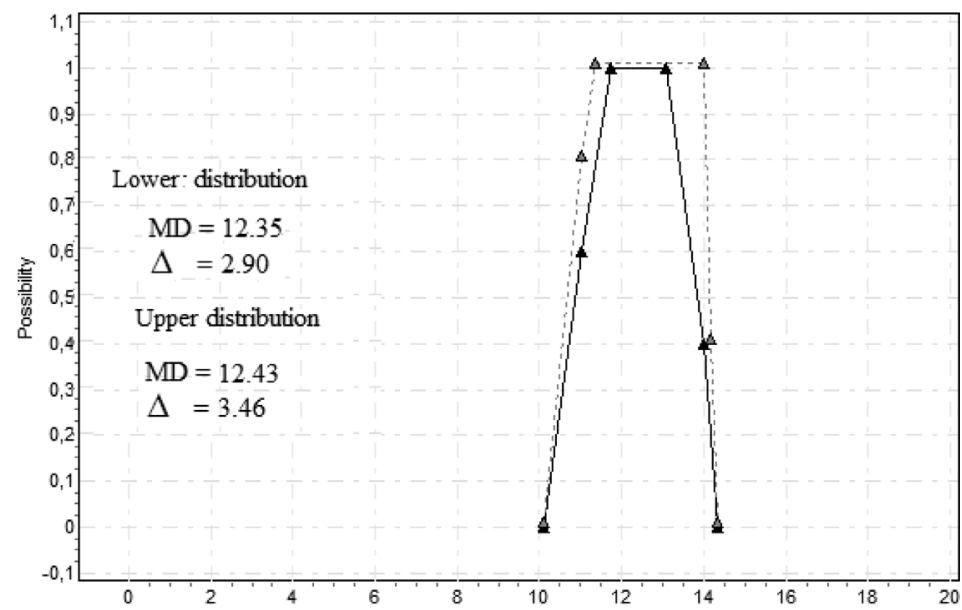

$\longrightarrow$ - sheltering - Lower distribution $-\cdots-\cdots \cdot \cdots$ sheltering - Upper distribution

Figure 18. Aggregated "Sheltering" distribution after Expert Kusto has twice changed his assessments.

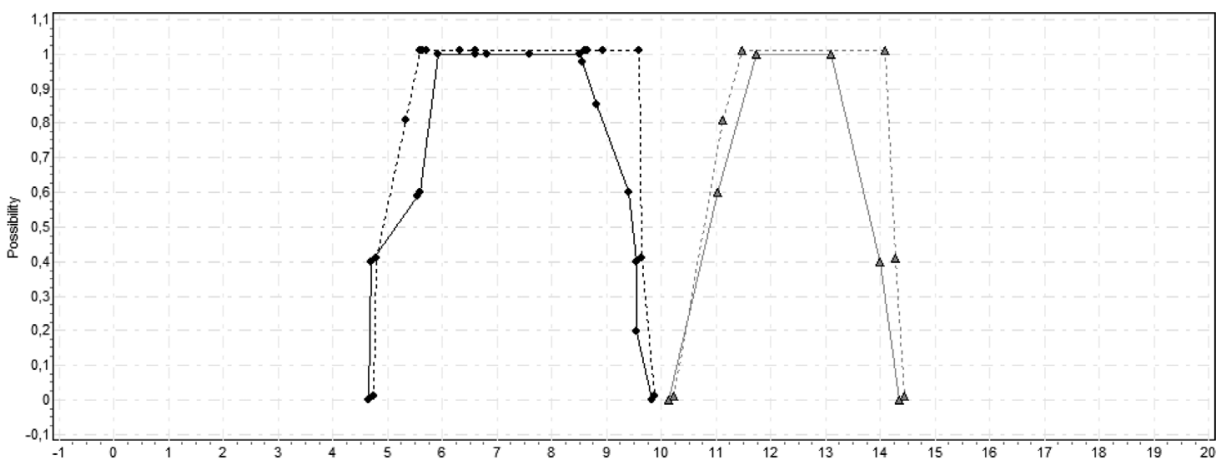

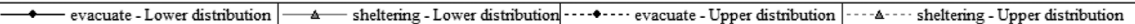

Figure 19. Comparison of the "Evacuate" and "Sheltering" alternatives.

relevant final decision. These proposals constitute a formal approach for increasing the reliability of the SA-assessment step whenever this assessment is based on several human sources of information. The key points of the proposed DSS are:

- the possibility distribution based representation that allows presenting the variability in imprecise assessments provided by experts, even when the assessments diverge (multimodal distribution);

- the nonlinear aggregation model allows taking into account both the relative importance of criteria and their interactions according to the head manager's preference model in an understandable framework for uncertainty management;

- extension of the notion of explanatory variables, as commonly used in statistical linear regression models, by defining the contribution to overall uncertainty. This definition proves useful in identifying the criteria on which the deliberation must focus. 
On the basis of this conceptual framework, a DSS has been developed to help the head manager of an emergency unit dedicated to a given road traffic accident situation. The case study has demonstrated that the proposed uncertainty representation of experts' assessments and their aggregation by a CI can provide the head manager with relevant information. During a subsequent step, the determination of contributions to overall uncertainty led to saving time in the final decision deliberation with experts, as well as improving the transparency and traceability of the decision in an easily comprehensive representation. Finally, participants' feedback has also revealed the need to enhance information visualization and interaction to simplify the sensitivity analysis.

\section{Acknowledgements}

The authors want to thank the French National Research Agency for financing the ISyCri project, its advices and relevant remarks on both theoretical and technical aspects during management reviews.

\section{References}

1. Van de Walle B, Turoff M. Handbook on decision support systems. Berlin, Germany: Springer; 2008. pp 295-316.

2. Li J, Li Q, Liu C, Khan SU, Ghani N. Community-based collaborative information system for emergency management. Comput Oper Res 2012; http://dx.doi.org/ 10.1016/j.cor.2012.03.018.

3. Burke E, Hendry C. Decision making on the London incident ground: An exploratory study. J Manag Psychol 1997;12(1):40-47.

4. Mendonça D. Decision support system for improvisation in response to extreme events: learning from the response to the 2001 World Trade Center Attack. Decis Support Syst 2007;43: 952-967.

5. Sayegh L, Anthony WP, Perrewé PL. Managerial decision-making under crisis: the role of emotion in an intuitive decision process. Human Res Manage Rev 2004;14:179-199.

6. Farinelli A, Nardi D, Pigliacampo R, Rossi M, Settembre GP. Cooperative Situation Assessment in a Maritime Scenario. Int J Intell Syst 2012;27(5):477-501.

7. Nokhbatolfoghahaayee H, Menhaj MB, Shafiee M. Fuzzy decision support system for crisis management with a new structure for decision making. Exp Syst Appl 2010;37:3545-3552.

8. Peng Y, Zhang Y, Tang Y, Li S. An incident information management framework based on data integration, data mining, and multi-criteria decision making. Decis Support Syst 2011;51:316-327.

9. Ju Y, Wang A. Emergency alternative evaluation under group decision makers: a method of incorporating DS/AHP with extended TOPSIS. Exp Syst Appl 2012;39:1315-1323.

10. Endsley MR. Measurement of situation awareness in dynamic systems. Hum Factors 1995;37(1):65-84.

11. Feng Y, Teng T, Tan A. Modelling situation awareness for context-aware decison support. Exp Syst Appl 2009;3:455-463.

12. Grabisch M, Roubens M. The application of fuzzy integrals in multi-criteria decision making. Eur J Oper Res 1996;89:445-456.

13. Yager RR. Criteria aggregations functions using fuzzy measures and the Choquet integral. Int J Fuzzy Sys 1999;1:96-112.

14. Dubois D, Prade H. Possibility theory: An approach to computerized processing of uncertainty. New York: Plenum Press; 1988.

15. Beynon M. DS/AHP method: A mathematical analysis, including an understanding of uncertainty. Eur J Oper Res 2002;140(1):148-164. 
16. Ma FM, Guo YJ, Shan X. Analysis of the Impact of Attitudinal Character on the Multicriteria Decision Making with OWA Operators. Int J Intell Syst 2012;27(5):502-518.

17. Ma W, Xiong W, Luo X. A model for Decision Making with Missing Imprecise, and Uncertain Evaluations of Multiple Criteria. Int J Intell Syst 2013;28(2):152-184.

18. Hartel CE, Smith K, Prince C. Defining aircrew coordination: Searching mishaps for meaning. In: Sixth Symp Aviation Psychology, Colombus, Ohio, 1991.

19. Endsley MR. Toward a theory of situation awareness in dynamic systems. Hum Factors 1995;37(1):32-64.

20. Curiel JM, Anhalt MD, Tran JJ, Yao KT. Developing Situation Awareness Metrics in a Synthetic Battlespace Environment. In: Interservice/Industry Training, Simulation, and Education Conference (I/ITSEC), 2005. pp 1451-1459.

21. Weick KE. Enacted sensemaking in crisis situation. J Manage Studies 1995;25(4):305-317.

22. Cheng $\mathrm{CH}$. Evaluating naval tactical missile systems by fuzzy AHP based on the grade value of membership function. Eur J Oper Res 1996;96:343-350.

23. Hsu HM, Chen CT. Aggregation of fuzzy opinions under group decision making. Fuzzy Sets and Syst 1996;79:279-285.

24. Lu C, Lan J, Wang Z. Aggregation of fuzzy opinions under decision-making based on similarity and distance. J Syst Sci Complexity 2006;19:63-71.

25. Dubois D, Prade H. Decision-making process, ISTE. New Jersey: Wiley; 2009. pp 85-156.

26. Grabisch M, Labreuche C. A decade of application of the Choquet and Sugeno integrals in multi-criteria decision aid. Ann Oper Res 2010;175(1):247-290.

27. Motro A. Sources of uncertainty in information systems. In: Second Workshop on Uncertainty Management and Information Systems: From Needs to Solutions, Catalina Island, CA, 1993.

28. Dubois D. Possibility theory and statistical reasoning. Comput Stat Data Anal 2006;51(1):47-69.

29. Denguir-Rekik A, Mauris G, Montmain J. A possibilistic-valued multi-criteria decisionmaking support for marketing activities in e-commerce: Feedback based diagnosis system. Eur J Oper Res 2009;195(3):876-888.

30. Dempster AP. Upper and Lower probabilities induced by multivalued mapping. Ann Math Stat 1967;38:325-339.

31. Shafer G. A mathematical theory of evidence. Princeton, NJ: Princeton University Press; 1976.

32. Mauris G, Lasserre V, Foulloy L. A fuzzy approach for the expression of uncertainty in measurement. Int J Meas 2001; 29(3):65-177.

33. Destercke S, Dubois D, Chojnacki E. Possibilistic information fusion using maximal coherent subsets. IEEE Trans Fuzzy Syst 2009;17:79-92.

34. Dubois D, Prade H. Fuzzy sets and statistical data. Eur J Oper Res 1986;25:345-356.

35. Dubois D, Prade H. A set-theoretic view of belief functions. Logical operations and approximations by fuzzy sets. Int J Gen Syst 1986;12(3):193-226.

36. Rescher N, Manor R. On inference from inconsistent premises. Theory Decis 1970;1:79219.

37. Dubois D, Prade H. The mean value of a fuzzy number. Fuzzy Sets Syst 1987;24:279-300.

38. Grabisch M. k-Ordered Discrete Fuzzy Measures and Their Representation. Fuzzy Sets Syst 1997;92:167-189.

39. Marichal JL. Aggregation operators. Heidelberg, Germany: Physica Verlag; 2002. pp 224-244.

40. Grabisch M, Labreuche C, Vansnick J. On the extension of pseudo Boolean function for the interacting bipolar criteria. Eur J Oper Res 2003;148:28-47.

41. Clivillé V, Berrah L, Mauris G. Quantitative expression and aggregation of performance measurements based on the Macbeth multi-criteria method. Int $\mathrm{J}$ Prod Econ 2007;105(1):171-189.

42. Montmain J, Mauris G, Akharraz A. Elucidation and Decisional Risk in a Multi Criteria Decision based on a Choquet Integral Aggregation-A Cybernetic Framework. Int J MultiCriteria Decis Anal 2005;13(5-6):239-258. 
43. Zadeh LA. Fuzzy Sets as a basis for a theory of possibility. Fuzzy Sets Syst 1978;1:3-28.

44. Grabisch M, Nguyen HT, Walker EA. Fundamentals of uncertainty calculi with applications to fuzzy inference. Dordrecht, the Netherlands: Kluwer Academic; 1994.

45. Denguir-Rekik A, Mauris G, Montmain J. Propagation of uncertainty by the possibility theory in a Choquet integral based Decision making for an E-commerce website choice support. IEEE Trans Instrum Meas 2006;55:721-728.

46. Benaben F, Hanachi C, Matthieu L, Couget P, Chapurlat V. A metamodel and its ontology to guide crisis characterization and its collaborative management. In: Fifth Int Conf on Information Systems for Crisis Response and Management, ISCRAM, Washington, USA, 2008.

47. Truptil S, Benaben F, Pingaud H. A mediation information system to help to coordinate a response to a crisis. In: Eleventh IFIP Working Conference on Virtual Enterprise, SaintEtienne, France; 2010. pp 173-180.

48. Bana e Costa C, Changas M. A career choice problem: An example of how to use Macbeth to build a quantitative value model based on qualitative value judgements. Eur J Oper Res 2004;153:323-331.

49. Bana e Costa C, De Corte JM, Vansnick JC. On the mathematical foundations of MACBETH. In: Figueira J, Greco S, Ehrgott M, editors. Multiple criteria decision analysis: State of the art surveys. New York: Springer Science + Business Media Inc.; 2004. pp 409-442. 